\title{
Adaptive Evolution of Social Traits: \\ Origin, Trajectories, and Correlations of Altruism and Mobility
}

Jean-François Le Galliard (j.f.I.galliard@ @io.uio.no)

Régis Ferrière (ferriere@ biologie.ens.fr)

Ulf Dieckmann (dieckmann @iiasa.ac.at)

\section{Approved by}

Leen Hordijk

Director, IIASA

March 2005 Institute, its National Member Organizations, or other organizations supporting the work. 


\section{IIASA STUDIES IN ADAPTIVE DYNAMICS No. 94}

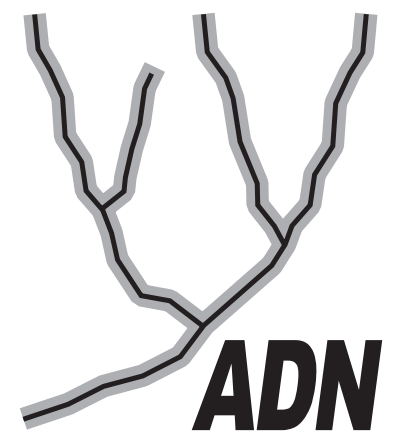

The Adaptive Dynamics Network at IIASA fosters the development of new mathematical and conceptual techniques for understanding the evolution of complex adaptive systems.

Focusing on these long-term implications of adaptive processes in systems of limited growth, the Adaptive Dynamics Network brings together scientists and institutions from around the world with IIASA acting as the central node.

Scientific progress within the network is collected in the IIASA Studies in Adaptive Dynamics series.

No. 1 Metz JAJ, Geritz SAH, Meszéna G, Jacobs FJA, van Heerwaarden JS: Adaptive Dynamics: A Geometrical Study of the Consequences of Nearly Faithful Reproduction. IIASA Working Paper WP-95-099 (1995). van Strien SJ, Verduyn Lunel SM (eds): Stochastic and Spatial Structures of Dynamical Systems, Proceedings of the Royal Dutch Academy of Science (KNAW Verhandelingen), North Holland, Amsterdam, pp. 183-231 (1996).

No. 2 Dieckmann U, Law R: The Dynamical Theory of Coevolution: A Derivation from Stochastic Ecological Processes. IIASA Working Paper WP-96-001 (1996). Journal of Mathematical Biology 34:579-612 (1996).

No. 3 Dieckmann U, Marrow P, Law R: Evolutionary Cycling of Predator-PreyInteractions: Population Dynamics and the Red Queen. IIASA Preprint (1995). Journal of Theoretical Biology 176:91-102 (1995).

No. 4 Marrow P, Dieckmann U, Law R: Evolutionary Dynamics of Predator-Prey Systems: An Ecological Perspective. IIASA Working Paper WP-96-002 (1996). Journal of Mathematical Biology 34:556-578 (1996).

No. 5 Law R, Marrow P, Dieckmann U: On Evolution under Asymmetric Competition. IIASA Working Paper WP-96-003 (1996). Evolutionary Ecology 11:485-501 (1997).

No. 6 Metz JAJ, Mylius SD, Diekmann O: When Does Evolution Optimize? On the Relation Between Types of Density Dependence and Evolutionarily Stable Life History Parameters. IIASA Working Paper WP-96-004 (1996).

No. 7 Ferrière R, Gatto M: Lyapunov Exponents and the Mathematics of Invasion in Oscillatory or Chaotic Populations. Theoretical Population Biology 48:126-171 (1995).

No. 8 Ferrière R, Fox GA: Chaos and Evolution. IIASA Preprint (1996). Trends in Ecology and Evolution 10:480485 (1995).

No. 9 Ferrière R, Michod RE: The Evolution of Cooperation in Spatially Heterogeneous Populations. IIASA Working Paper WP-96-029 (1996). The American Naturalist 147:692717 (1996).

No. 10 van Dooren TJM, Metz JAJ: Delayed Maturation in Temporally Structured Populations with Non-Equilibrium Dynamics. IIASA Working Paper WP-96-070 (1996). Journal of Evolutionary Biology 11:41-62 (1998).
No. 11 Geritz SAH, Metz JAJ, Kisdi É, Meszéna G: The Dynamics of Adaptation and Evolutionary Branching. IIASA Working Paper WP-96-077 (1996). Physical Review Letters 78:2024-2027 (1997).

No. 12 Geritz SAH, Kisdi É, Meszéna G, Metz JAJ: Evolutionary Singular Strategies and the Adaptive Growth and Branching of the Evolutionary Tree. IIASA Working Paper WP-96-114 (1996). Evolutionary Ecology 12:35-57 (1998).

No. 13 Heino M, Metz JAJ, Kaitala V: Evolution of Mixed Maturation Strategies in Semelparous Life-Histories: The Crucial Role of Dimensionality of Feedback Environment. IIASA Working Paper WP-96-126 (1996). Philosophical Transactions of the Royal Society of London Series B 352:1647-1655 (1997).

No. 14 Dieckmann U: Can Adaptive Dynamics Invade? IIASA Working Paper WP-96-152 (1996). Trends in Ecology and Evolution 12:128-131 (1997).

No. 15 Meszéna G, Czibula I, Geritz SAH: Adaptive Dynamics in a 2-Patch Environment: A Simple Model for Allopatric and Parapatric Speciation. IIASA Interim Report IR-97-001 (1997). Journal of Biological Systems 5:265-284 (1997).

No. 16 Heino M, Metz JAJ, Kaitala V: The Enigma of Frequency-Dependent Selection. IIASA Interim Report IR97-061 (1997). Trends in Ecology and Evolution 13:367-370 (1998).

No. 17 Heino M: Management of Evolving Fish Stocks. IIASA Interim Report IR-97-062 (1997). Canadian Journal of Fisheries and Aquatic Sciences 55:1971-1982 (1998).

No. 18 Heino M: Evolution of Mixed Reproductive Strategies in Simple Life-History Models. IIASA Interim Report IR-97063 (1997).

No. 19 Geritz SAH, van der Meijden E, Metz JAJ: Evolutionary Dynamics of Seed Size and Seedling Competitive Ability. IIASA Interim Report IR-97-071 (1997). Theoretical Population Biology 55:324-343 (1999).

No. 20 Galis F, Metz JAJ: Why Are There So Many Cichlid Species? On the Interplay of Speciation and Adaptive Radiation. IIASA Interim Report IR-97-072 (1997). Trends in Ecology and Evolution 13:1-2 (1998). 
No. 21 Boerlijst MC, Nowak MA, Sigmund K: Equal Pay for all Prisoners/ The Logic of Contrition. IIASA Interim Report IR-97-073 (1997). American Mathematical Society Monthly 104:303-307 (1997). Journal of Theoretical Biology 185:281-293 (1997).

No. 22 Law R, Dieckmann U: Symbiosis Without Mutualism and the Merger of Lineages in Evolution. IIASA Interim Report IR-97-074 (1997). Proceedings of the Royal Society of London Series B 265:1245-1253 (1998).

No. 23 Klinkhamer PGL, de Jong TJ, Metz JAJ: Sex and Size in Cosexual Plants. IIASA Interim Report IR-97-078 (1997). Trends in Ecology and Evolution 12:260-265 (1997).

No. 24 Fontana W, Schuster P: Shaping Space: The Possible and the Attainable in RNA Genotype-Phenotype Mapping. IIASA Interim Report IR-98-004 (1998). Journal of Theoretical Biology 194:491-515 (1998).

No. 25 Kisdi É, Geritz SAH: Adaptive Dynamics in Allele Space: Evolution of Genetic Polymorphism by Small Mutations in a Heterogeneous Environment. IIASA Interim Report IR-98-038 (1998). Evolution 53:993-1008 (1999).

No. 26 Fontana W, Schuster P: Continuity in Evolution: On the Nature of Transitions. IIASA Interim Report IR-98-039 (1998). Science 280:1451-1455 (1998).

No. 27 Nowak MA, Sigmund K: Evolution of Indirect Reciprocity by Image Scoring/ The Dynamics of Indirect Reciprocity. IIASA Interim Report IR-98-040 (1998). Nature 393:573-577 (1998). Journal of Theoretical Biology 194:561574 (1998).

No. 28 Kisdi É: Evolutionary Branching Under Asymmetric Competition. IIASA Interim Report IR-98-045 (1998). Journal of Theoretical Biology 197:149-162 (1999).

No. 29 Berger U: Best Response Adaptation for Role Games. IIASA Interim Report IR-98-086 (1998).

No. 30 van Dooren TJM: The Evolutionary Ecology of Dominance-Recessivity. IIASA Interim Report IR-98-096 (1998). Journal of Theoretical Biology 198:519-532 (1999).

No. 31 Dieckmann U, O'Hara B, Weisser W: The Evolutionary Ecology of Dispersal. IIASA Interim Report IR-98-108 (1998). Trends in Ecology and Evolution 14:88-90 (1999).

No. 32 Sigmund K: Complex Adaptive Systems and the Evolution of Reciprocation. IIASA Interim Report IR-98-100 (1998). Ecosystems 1:444-448 (1998).

No. 33 Posch M, Pichler A, Sigmund K: The Efficiency of Adapting Aspiration Levels. IIASA Interim Report IR-98103 (1998). Proceedings of the Royal Society London Series B 266:1427-1435 (1999).

No. 34 Mathias A, Kisdi É: Evolutionary Branching and Coexistence of Germination Strategies. IIASA Interim Report IR-99-014 (1999).

No. 35 Dieckmann U, Doebeli M: On the Origin of Species by Sympatric Speciation. IIASA Interim Report IR-99-013 (1999). Nature 400:354-357 (1999).

No. 36 Metz JAJ, Gyllenberg M: How Should We Define Fitness in Structured Metapopulation Models? Including an Application to the Calculation of Evolutionarily Stable Dispersal Strategies. IIASA Interim Report IR-99-019 (1999). Proceedings of the Royal Society of London Series B 268:499508 (2001)
No. 37 Gyllenberg M, Metz JAJ: On Fitness in Structured Metapopulations. IIASA Interim Report IR-99-037 (1999). Journal of Mathematical Biology 43:545-560 (2001).

No. 38 Meszéna G, Metz JAJ: Species Diversity and Population Regulation: The Importance of Environmental Feedback Dimensionality. IIASA Interim Report IR-99-045 (1999).

No. 39 Kisdi É, Geritz SAH: Evolutionary Branching and Sympatric Speciation in Diploid Populations. IIASA Interim Report IR-99-048 (1999).

No. 40 Ylikarjula J, Heino M, Dieckmann U: Ecology and Adaptation of Stunted Growth in Fish. IIASA Interim Report IR-99-050 (1999). Evolutionary Ecology 13:433-453 (1999).

No. 41 Nowak MA, Sigmund K: Games on Grids. IIASA Interim Report IR-99-038 (1999). Dieckmann U, Law R, Metz JAJ (eds): The Geometry of Ecological Interactions: Simplifying Spatial Complexity, Cambridge University Press, Cambridge, UK, pp. 135-150 (2000).

No. 42 Ferrière R, Michod RE: Wave Patterns in Spatial Games and the Evolution of Cooperation. IIASA Interim Report IR-99-041 (1999). Dieckmann U, Law R, Metz JAJ (eds): The Geometry of Ecological Interactions: Simplifying Spatial Complexity, Cambridge University Press, Cambridge, UK, pp. 318-332 (2000).

No. 43 Kisdi É, Jacobs FJA, Geritz SAH: Red Queen Evolution by Cycles of Evolutionary Branching and Extinction. IIASA Interim Report IR-00-030 (2000). Selection 2:161176 (2001).

No. 44 Meszéna G, Kisdi É, Dieckmann U, Geritz SAH, Metz JAJ: Evolutionary Optimisation Models and Matrix Games in the Unified Perspective of Adaptive Dynamics. IIASA Interim Report IR-00-039 (2000). Selection 2:193-210 (2001).

No. 45 Parvinen K, Dieckmann U, Gyllenberg M, Metz JAJ: Evolution of Dispersal in Metapopulations with Local Density Dependence and Demographic Stochasticity. IIASA Interim Report IR-00-035 (2000). Journal of Evolutionary Biology $16: 143-153$ (2003).

No. 46 Doebeli M, Dieckmann U: Evolutionary Branching and Sympatric Speciation Caused by Different Types of Ecological Interactions. IIASA Interim Report IR-00-040 (2000). The American Naturalist 156:S77-S101 (2000).

No. 47 Heino M, Hanski I: Evolution of Migration Rate in a Spatially Realistic Metapopulation Model. IIASA Interim Report IR-00-044 (2000). The American Naturalist 157:495511 (2001).

No. 48 Gyllenberg M, Parvinen K, Dieckmann U: Evolutionary Suicide and Evolution of Dispersal in Structured Metapopulations. IIASA Interim Report IR-00-056 (2000). Journal of Mathematical Biology 45:79-105 (2002).

No. 49 van Dooren TJM: The Evolutionary Dynamics of Direct Phenotypic Overdominance: Emergence Possible, Loss Probable. IIASA Interim Report IR-00-048 (2000). Evolution 54: 1899-1914 (2000).

No. 50 Nowak MA, Page KM, Sigmund K: Fairness Versus Reason in the Ultimatum Game. IIASA Interim Report IR00-57 (2000). Science 289:1773-1775 (2000).

No. 51 de Feo O, Ferrière R: Bifurcation Analysis of Population Invasion: On-Off Intermittency and Basin Riddling. IIASA Interim Report IR-00-074 (2000). International Journal of Bifurcation and Chaos 10:443-452 (2000). 
No. 52 Heino M, Laaka-Lindberg S: Clonal Dynamics and Evolution of Dormancy in the Leafy Hepatic Lophozia Silvicola. IIASA Interim Report IR-01-018 (2001). Oikos 94:525-532 (2001).

No. 53 Sigmund K, Hauert C, Nowak MA: Reward and Punishment in Minigames. IIASA Interim Report IR-01-031 (2001). Proceedings of the National Academy of Sciences of the USA 98:10757-10762(2001).

No. 54 Hauert C, De Monte S, Sigmund K, Hofbauer J: Oscillations in Optional Public Good Games. IIASA Interim Report IR-01-036 (2001).

No. 55 Ferrière R, Le Galliard J: Invasion Fitness and Adaptive Dynamics in Spatial Population Models. IIASA Interim Report IR-01-043 (2001). Clobert J, Dhondt A, Danchin E, Nichols J (eds): Dispersal, Oxford University Press, pp. 57-79 (2001).

No. 56 de Mazancourt C, Loreau M, Dieckmann U: Can the Evolution of Plant Defense Lead to Plant-Herbivore Mutualism. IIASA Interim Report IR-01-053 (2001). The American Naturalist 158: 109-123 (2001).

No. 57 Claessen D, Dieckmann U: Ontogenetic Niche Shifts and Evolutionary Branching in Size-Structured Populations. IIASA Interim Report IR-01-056 (2001). Evolutionary Ecology Research 4:189-217 (2002).

No. 58 Brandt H: Correlation Analysis of Fitness Landscapes. IIASA Interim Report IR-01-058 (2001).

No. 59 Dieckmann U: Adaptive Dynamics of Pathogen-Host Interacations. IIASA Interim Report IR-02-007 (2002). Dieckmann U, Metz JAJ, Sabelis MW, Sigmund K (eds): Adaptive Dynamics of Infectious Diseases: In Pursuit of Virulence Management, Cambridge University Press, Cambridge, UK, pp. 39-59 (2002).

No. 60 Nowak MA, Sigmund K: Super- and Coinfection: The Two Extremes. IIASA Interim Report IR-02-008 (2002). Dieckmann U, Metz JAJ, Sabelis MW, Sigmund K (eds): Adaptive Dynamics of Infectious Diseases: In Pursuit of Virulence Management, Cambridge University Press, Cambridge, UK, pp. 124-137 (2002).

No. 61 Sabelis MW, Metz JAJ: Perspectives for Virulence Management: Relating Theory to Experiment. IIASA Interim Report IR-02-009 (2002). Dieckmann U, Metz JAJ, Sabelis MW, Sigmund K (eds): Adaptive Dynamics of Infectious Diseases: In Pursuit of Virulence Management, Cambridge University Press, Cambridge, UK, pp. 379-398 (2002).

No. 62 Cheptou P, Dieckmann U: The Evolution of SelfFertilization in Density-Regulated Populations . IIASA Interim Report IR-02-024 (2002). Proceedings of the Royal Society of London Series B 269:1177-1186 (2002).

No. 63 Bürger R: Additive Genetic Variation Under Intraspecific Competition and Stabilizing Selection: A Two-Locus Study. IIASA Interim Report IR-02-013 (2002). Theoretical Population Biology 61:197-213 (2002).

No. 64 Hauert C, De Monte S, Hofbauer J, Sigmund K: Volunteering as Red Queen Mechanism for Co-operation in Public Goods Games. IIASA Interim Report IR-02-041 (2002). Science 296:1129-1132(2002).

No. 65 Dercole F, Ferrière R, Rinaldi S: Ecological Bistability and Evolutionary Reversals under Asymmetrical Competition. IIASA Interim Report IR-02-053 (2002). Evolution 56:1081-1090 (2002).
No. 66 Dercole F, Rinaldi S: Evolution of Cannibalistic Traits: Scenarios Derived from Adaptive Dynamics. IIASA Interim Report IR-02-054 (2002). Theoretical Population Biology 62:365-374 (2002).

No. 67 Bürger R, Gimelfarb A: Fluctuating Environments and the Role of Mutation in Maintaining Quantitative Genetic Variation. IIASA Interim Report IR-02-058 (2002). Genetical Research 80:31-46 (2002).

No. 68 Bürger R: On a Genetic Model of Intraspecific Competition and Stabilizing Selection. IIASA Interim Report IR02-062 (2002). Amer. Natur. 160:661-682 (2002).

No. 69 Doebeli M, Dieckmann U: Speciation Along Environmental Gradients. IIASA Interim Report IR-02-079 (2002). Nature 421:259-264 (2003).

No. 70 Dercole F, Irisson J, Rinaldi S: Bifurcation Analysis of a Prey-Predator Coevolution Model. IIASA Interim Report IR-02-078 (2002). SIAM Journal on Applied Mathematics 63:1378-1391 (2003).

No. 71 Le Galliard J, Ferrière R, Dieckmann U: The Adaptive Dynamics of Altruism in Spatially Heterogeneous Populations. IIASA Interim Report IR-03-006 (2003). Evolution 57:1-17 (2003).

No. 72 Taborsky B, Dieckmann U, Heino M: Unexpected Discontinuities in Life-History Evolution under SizeDependent Mortality. IIASA Interim Report IR-03-004 (2003). Proceedings of the Royal Society of London Series B 270:713-721 (2003).

No. 73 Gardmark A, Dieckmann U, Lundberg P: LifeHistory Evolution in Harvested Populations: The Role of Natural Predation. IIASA Interim Report IR-03-008 (2003). Evolutionary Ecology Research 5:239-257 (2003).

No. 74 Mizera F, Meszéna G: Spatial Niche Packing, Character Displacement and Adaptive Speciation Along an Environmental Gradient. IIASA Interim Report IR-03-062 (2003). Evolutionary Ecology Research 5: 363-382 (2003).

No. 75 Dercole F: Remarks on Branching-Extinction Evolutionary Cycles. IIASA Interim Report IR-03-075 (2003). Journal of Mathematical Biology 47: 569-580 (2003).

No. 76 Hofbauer J, Sigmund K: Evolutionary Game Dynamics. IIASA Interim Report IR-03-078 (2003). Bulletin of the American Mathematical Society 40: 479-519 (2003).

No. 77 Ernande B, Dieckmann U, Heino M: Adaptive Changes in Harvested Populations: Plasticity and Evolution of Age and Size at Maturation. IIASA Interim Report IR03-058 (2003). Proceedings of the Royal Society of London Series B-Biological Sciences, 271: 415-423 (2004).

No. 78 Hanski I, Heino M: Metapopulation-Level Adaptation of Insect Host Plant Preference and Extinction-Colonization Dynamics in Heterogeneous Landscapes. IIASA Interim Report IR-03-028 (2003). Theoretical Population Biology 63:309-338 (2003).

No. 79 van Doorn G, Dieckmann U, Weissing FJ: Sympatric Speciation by Sexual Selection: A Critical Re-Evaluation. IIASA Interim Report IR-04-003 (2004). American Naturalist 163: 709-725 (2004).

No. 80 Egas M, Dieckmann U, Sabelis MW: Evolution Restricts the Coexistence of Specialists and Generalists - the Role of Trade-off Structure. IIASA Interim Report IR-04-004 (2004). 
No. 81 Ernande B, Dieckmann U: The Evolution of Phenotypic Plasticity in Spatially Structured Environments: Implications of Intraspecific Competition, Plasticity Costs, and Environmental Characteristics. IIASA Interim Report IR-04-006 (2004). Journal of Evolutionary Biology 17 (3): 613-628 (2004).

No. 82 Cressman R, Hofbauer J: Measure Dynamics on a One-Dimensional Continuous Trait Space: Theoretical Foundations for Adaptive Dynamics. IIASA Interim Report IR04-016 (2004).

No. 83 Cressman R: Dynamic Stability of the Replicator Equation with Continuous Strategy Space. IIASA Interim Report IR-04-017 (2004).

No. 84 Ravigné V, Olivieri I, Dieckmann U: Implications of Habitat Choice for Protected Polymorphisms. IIASA Interim Report IR-04-005 (2004). Evolutionary Ecology Research 6: 125-145 (2004).

No. 85 Nowak MA, Sigmund K: Evolutionary Dynamics of Biological Games. IIASA Interim Report IR-04-013 (2004). Science 303: 793-799 (2004).

No. 86 Vukics A, Asbóth J, Meszéna G: Speciation in Multidimensional Evolutionary Space. IIASA Interim Report IR-04-028 (2004). Physical Review E 684 (2003).

No. 87 de Mazancourt C, Dieckmann U: Trade-off Geometries and Frequency-dependent Selection. IIASA Interim Report IR-04-039 (2004).
No. 88 Cadet CR, Metz JAJ, Klinkhamer PGL: Size and the Not-So-Single Sex: disentangling the effects of size on sex allocation. IIASA Interim Report IR-04-084 (2004). American Naturalist, 164: 779-792 (2004).

No. 89 Rueffler C, van Dooren TJM, Metz JAJ: Adaptive Walks on Changing Landscapes: Levins' Approach Extended. IIASA Interim Report IR-04-083 (2004). Theoretical Population Biology, 65: 165-178 (2004).

No. 90 de Mazancourt C, Loreau M, Dieckmann U: Understanding Mutualism When There is Adaptation to the Partner. IIASA Interim Report IR-05-016 (2005).

No. 91 Dieckmann U, Doebeli M: Pluralism in Evolutionary Theory. IIASA Interim Report IR-05-017 (2005).

No. 92 Doebeli M, Dieckmann U, Metz JAJ, Tautz D: What We Have Also Learned. IIASA Interim Report IR-05-018 (2005).

No. 93 Egas M, Sabelis MW, Dieckmann U: Evolution of Specialization and Ecological Character Displacement of Herbivores Along a Gradient of Plant Quality. IIASA Interim Report IR-05-019 (2005).

No. 94 Le Galliard J, Ferrière R, Dieckmann U: Adaptive Evolution of Social Traits: Origin, Trajectories, and Correlations of Altruism and Mobility. IIASA Interim Report IR05-020 (2005).

Issues of the IIASA Studies in Adaptive Dynamics series can be obtained at www.iiasa.ac.at/Research/ADN/Series.html or by writing to adn@iiasa.ac.at. 


\section{Contents}

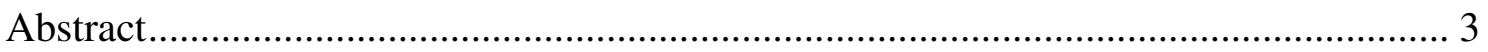

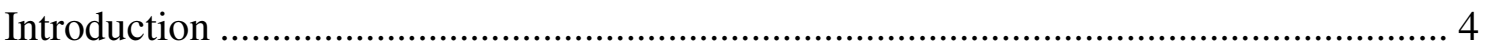

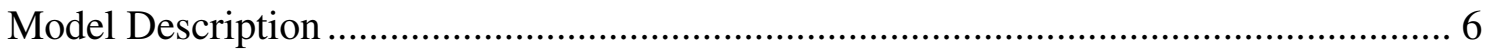

Population dynamics on social networks .................................................................. 7

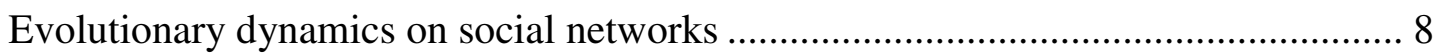

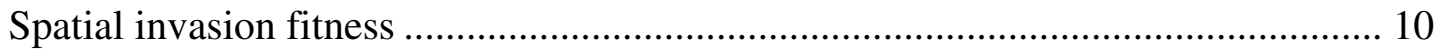

Selective Pressures on Mobility and Altruism ............................................................. 13

Separate Evolution of Mobility and Altruism ........................................................... 17

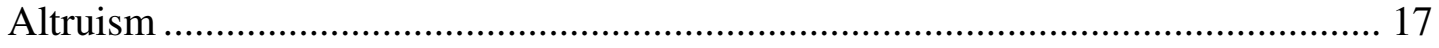

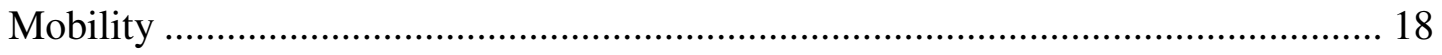

Joint Evolution of Altruism and Mobility .................................................................. 20

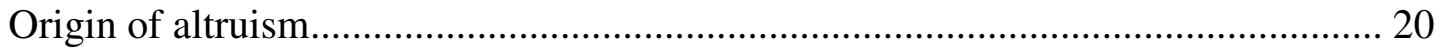

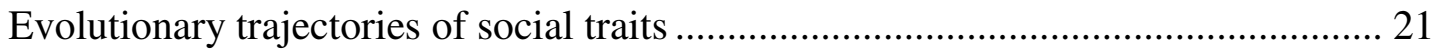

Evolutionarily induced correlations between altruism and mobility ........................ 22

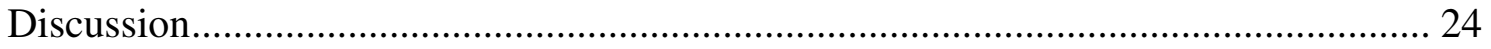

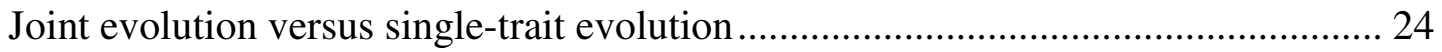

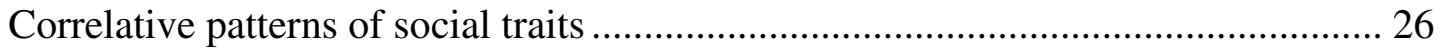

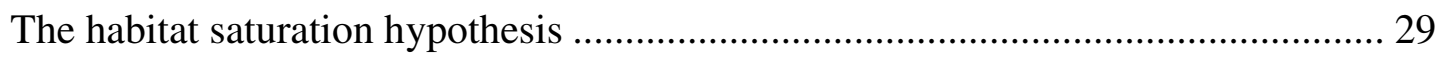

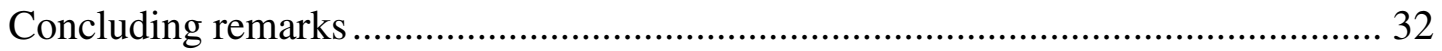

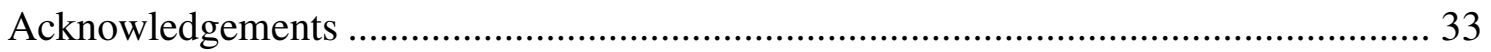

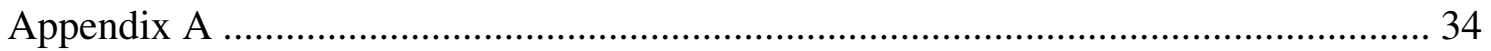

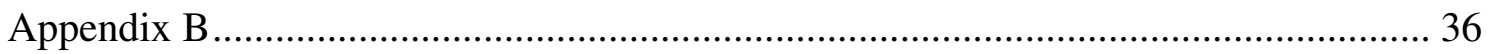

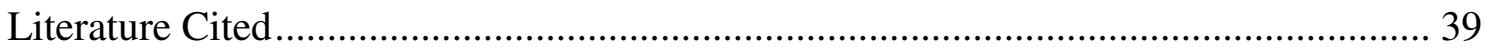

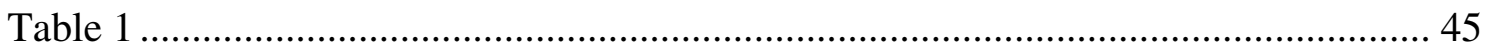

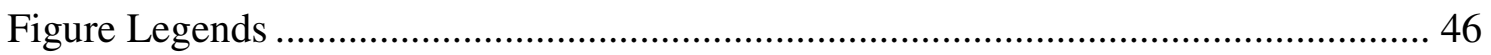

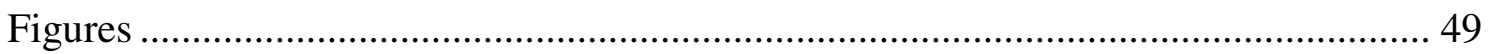



CORRELATIONS OF ALTRUISM AND MOBILITY

5 Jean-François Le Galliard

6 Fonctionnement et évolution des systèmes écologiques, CNRS UMR 7625

7 Ecole Normale Supérieure, 75005 Paris, France

8 Present address: Center for Ecological and Evolutionary Synthesis, University of Oslo P.O. Box 1050, Blindern, Oslo NO-0316, Norway E-mail: j.f.l.galliard@bio.uio.no

\section{Régis Ferrière}

13 1) Fonctionnement et évolution des systèmes écologiques, CNRS UMR 7625

14 Ecole Normale Supérieure, 75005 Paris, France

15 E-mail: ferriere@biologie.ens.fr

16 2) Department of Ecology and Evolutionary Biology

17 University of Arizona, Tucson AZ 85721, USA

18

\section{Ulf Dieckmann}

20 Adaptive Dynamics Network, International Institute for Applied Systems Analysis

21 Schlossplatz 1, A-2361 Laxenburg, Austria

22 E-mail: dieckmann@iiasa.ac.at 
1 Keywords

2 Evolutionary dynamics, spatial invasion fitness, altruism, mobility, habitat saturation, kin selec3 tion.

4

$5 \quad$ Running head

$6 \quad$ Evolution of altruism and mobility.

7

8 List of elements to appear in the Expanded Online Edition

$9 \quad$ Online Appendix A

$10 \quad$ Online Appendix B 


\section{ABSTRACT}

2 Social behavior involves "staying and helping", two individual attributes that vary considerably 3 among organisms. Investigating the ultimate causes of such variation, this study integrates previ-

4 ously separate lines of research by analyzing the joint evolution of altruism and mobility. We 5 unfold the network of selective pressures and derive how these depend on physiological costs, 6 eco-evolutionary feedbacks, and a complex interaction between the evolving traits. Our analysis 7 highlights habitat saturation, both around individuals (local aggregation) and around unoccupied 8 space (local contention), as the key mediator of altruism and mobility evolution. Once altruism 9 and mobility are allowed to evolve jointly, three general insights emerge. First, the cost of mobil10 ity affects the origin of altruism, determining whether and how quickly selfishness is overcome.

11 Second, the cost of altruism determines which of two qualitatively different routes to sociality are 12 taken: an evolutionary reduction of mobility, resulting in higher habitat saturation, is either pre13 ceded or followed by the adaptive rise of altruism. Third, contrary to conventional expectations, a 14 positive correlation between evolutionarily stable levels of altruism and mobility can arise; this is 15 expected when comparing populations that evolved under different constraints on mobility or that 16 differ in other life history traits. 


\section{INTRODUCTION}

2 Sociality is an essential characteristic of life. It involves specific individual behaviors that lead to

3 the emergence of collective properties, new levels of natural selection, and the adaptive com-

4 plexification of living systems (Michod 1999). One of the intriguing features of sociality is that it 5 causes a double cost to individuals. Sociality typically requires, first, some form of altruistic be-

6 havior through which individuals sacrifice their own fitness for the benefit of others (Hamilton

7 1964a, b), and second, some reduction in individual mobility, allowing for sustained interaction,

8 which exacerbates competition for local resources (Frank 1995; Perrin and Lehmann 2001). The

9 benefits associated with these costs must be substantial enough that the involved genes are not

10 eliminated by natural selection. Thus, one of the challenges facing evolutionary theory is to ex-

11 plain the role of adaptive evolution in molding individual altruism along with the underlying

12 population structure, to help us understand the wide diversity of social systems observed in the

13 wild (Choe and Crespi 1997; Crespi 2001).

14 The double cost of sociality reflects only some of the selective pressures acting on social

15 traits. Low individual mobility may increase genetic relatedness between interacting individuals,

16 thus promoting inbreeding as well as the evolution of helping behaviors through kin selection

17 (Hamilton 1964b). Yet, the enhancement of neighbors' performance through altruistic interaction

18 may induce also habitat saturation and thus exacerbate local competition among kin (Grafen

19 1984; Queller 1992). Increased competition between relatives for local resources can in turn re-

20 duce or even totally negate the indirect genetic benefits of altruism (Taylor 1992; Wilson et al.

21 1992). The deleterious effects of kin competition resulting from low mobility have been demon-

22 strated in a recent comparative study of social traits in fig wasps. In these insects, strict philopatry 
1 of males competing for mates results in extremely strong local competition, which nullifies any

2 indirect genetic benefits of decreasing aggressiveness toward relatives (West et al. 2001). In other

3 social insects, limited dispersal can lead to competition between colonies founded by relatives

4 (Thorne 1997). In cooperatively breeding vertebrates, local recruitment can also cause competi-

5 tion among relatives for dominance and breeding opportunities within a group (Clutton-Brock

6 2002). In general, the balance between kin cooperation and kin competition affecting the evolu-

7 tion of altruism is bound to vary with the species' life history profile, the spatial scale over which

8 cooperation and competition occur, and the underlying habitat structure (Kelly 1994; Queller 9 1992).

10 In a recent study, Le Galliard et al. (2003) presented the analysis of a model accounting

11 for population viscosity (limited dispersal of offspring at birth) combined with adult mobility,

12 overlapping generations, and fluctuations in local population size caused by local interactions and

13 demographic stochasticity. In that model, the costs of local competition do not completely negate

14 the benefits kin cooperation - a finding echoed by other recent theoretical analyses (Irwin and

15 Taylor 2001; Mitteldorf and Wilson 2000). That study also highlighted the critical influence indi-

16 vidual mobility exerts on the evolution of altruism: high altruism could evolve only in species

17 with low mobility, whereas the evolutionary trajectory of highly mobile species was halted in a

18 state of 'quasi-selfishness'. However, the assumption (made in that and many other studies) of

19 mobility being fixed is appropriate only if mobility is strongly constrained by the environment or

20 the genetic system. Otherwise, mobility and altruism will be entangled in joint evolution: costs

21 and benefits of altruism depend on local spatial structures and thus on mobility (Ferrière and Le

22 Galliard 2001; Perrin and Lehmann 2001), while costs and benefits of mobility depend on the 23 amount of help on offer, as well as on habitat saturation, which are both affected by altruism 
1 (Emlen 1997; Helms Cahan et al. 2002). The purpose of the present study is to develop a unifying

2 approach to address this fundamental feedback in the evolution of sociality.

Extending the framework used by Le Galliard et al. (2003), we study the joint evolution of

4 altruism and mobility in a model in which individuals move and interact locally on a network of

5 suitable sites (Matsuda et al. 1992; van Baalen 2000). The notion of fitness that is appropriate for

6 characterizing frequency-dependent selection as it occurs in such a model is invasion fitness, that

7 is, the per capita growth rate of a mutant when rare in the environment set by the wild-type popu-

8 lation (Metz et al. 1992). This notion has been found to extend to kin selection processes

9 involving diallelic, haploid genetics (Frank 1998). Extending work by van Baalen and Rand

10 (1998), we derive invasion fitness from a set of correlation equations describing the population's

11 spatial structure (Ferrière and Le Galliard 2001; Le Galliard et al. 2003). On this basis, we then

12 (i) deduce the selective pressures acting on altruism and mobility traits, and relate these pressures

13 to the model's underlying parameters; (ii) analyze the trajectories of the joint evolution of these

14 traits, and their interplay with the population's spatial structure; and (iii) make predictions about

15 the correlation patterns between altruism and mobility induced by evolution in response to varia-

16 tion of life history traits or to ecological constraints across species or populations.

\section{MODEL DESCRIPTION}

18 In this model, interactions and mobility are local processes, occurring between neighboring sites

19 of a social network. Altruism and mobility are quantitative characters affecting the demographic

20 parameters of individuals. The resulting individual-based dynamics mold the local population

21 structure, from which the selective pressures on altruism and mobility arise. These pressures, in 
1 turn, determine the evolutionary outcomes we aim to understand. All parameters and variables

2 are listed in Table 1.

\section{Population dynamics on social networks}

4 Individuals are distributed over a network of sites. Each site may be empty or occupied by one

5 individual, and is randomly connected to $n$ other sites that define a neighborhood; $n$ is a fixed

6 parameter measuring the neighborhood size, or 'habitat connectivity'. Such spatial structure is

7 used classically to study social interactions (e.g., Rand 1998) and is typical of e.g. some verte-

8 brates that defend territories and move primarily among adjacent sites. We use a continuous-time

9 model in which generations overlap. During any small time interval, an individual may move to

10 an empty site within its neighborhood, or produce an offspring that is placed in an empty

11 neighboring site, or die. The population is 'viscous' (Hamilton 1964a, b) in the sense that off-

12 spring may be laid only in sites neighboring a parent's (not farther off); yet mobility is permitted

13 at any age, unconditionally to the occurrence of birth events. Thus, our notion of mobility differs

14 from that of 'natal dispersal' but is similar to 'breeding dispersal', which refers to an adult mov-

15 ing between different breeding sites. The per capita mobility rate $m$ and death rate $d$ are

16 unaffected by local interactions. Mobility is costly to individuals, with a negative effect on the

17 individual's intrinsic birth rate (Cohen and Motro 1989). The cost of mobility linearly impacts the

18 intrinsic birth rate such that the net per capita birth rate (in the absence of interaction) is given by

$19 b-v m$, where $b$ measures the intrinsic per capita birth rate of sessile organisms, and $v$ measures

20 the cost sensitivity to the mobility rate.

21 Two types of local density-dependent factors affect movement and reproduction. First, both

22 events are conditional on the availability of a neighboring empty site: consequently local crowd-

23 ing negatively affects the rates of mobility and birth. Second, reproduction is enhanced by 
1 altruistic interactions with neighbors, inducing a positive effect of local crowding. Thus, an altru-

2 istic donor improves the quality of the neighboring sites at the expense of its own reproduction,

3 as has been documented in some cooperatively breeding vertebrates (Cockburn 1998). In our

4 model, the altruism rate $u$ is defined by the per capita rate of energetic investment into altruistic

5 interactions. Altruistic behavior is directed evenly towards all neighboring sites, regardless of the

6 presence or phenotypes of neighbors. Consequently, every neighbor of a focal individual that

$7 \quad$ invests at rate $u$ into altruism sees her birth rate augmented by the amount $u / n$. We use the terms

8 'selfishness' and 'quasi-selfishness' to describe, respectively, phenotypes whose investment in

9 altruism is zero or nearly zero.

10 Typically, altruism carries a physiological cost. For example, adult suricates Suricata suri-

11 catta lose significant body weight during baby-sitting activities (Clutton-Brock et al. 1998). In

12 general, such a cost can depend on the level of altruistic investment in an accelerating, linear, or

13 decelerating way. With an accelerating cost, the marginal costs of altruism increase with the level

14 of altruism. Conversely, decelerating costs imply that increasing altruism at low levels is more

15 costly than at high levels. In the limiting case of linear costs, marginal costs are independent of

16 the level of altruism. These three patterns are captured by the expression $\kappa u^{\gamma}$, where $\kappa$ scales the

17 cost sensitivity to the altruism rate, and $\gamma$ determines whether costs are accelerating $(\gamma>1)$, linear

$18(\gamma=1)$, or decelerating $(\gamma<1)$. The combined cost of mobility and altruism diminishing the birth

19 rate is given by $C(m, u)=v m+\kappa u^{\gamma}$.

\section{Evolutionary dynamics on social networks}

21 The two traits evolving in our model are the altruism rate $u$ and the mobility rate $m$. Mutations,

22 which occur with a fixed probability $k$ per birth event, cause these rates to differ between off- 
1 spring and parent. Increments or decrements resulting from mutations are drawn randomly from a

2 normal probability distribution with zero mean, variance $\sigma^{2}$ (identical for both traits), and without

3 genetic correlations. Like in Le Galliard et al. (2003), we used the minimal process method

4 (Gillespie 1976) to simulate the evolutionary process on a social network of 900 sites, generated

5 by randomizing the edges of a $30 \times 30$ regular lattice with von Neumann neighborhoods and pe-

6 riodic boundaries.

In a large population in which mutations are rare and mutational steps are small, the sto-

8 chastic mutation-selection process described above can be approximated by a deterministic 9 process whose trajectories are solution of the canonical equation of adaptive dynamics 10 (Dieckmann and Law 1996) applied to this model,

11

$$
\frac{d}{d t}\left(\begin{array}{l}
m_{x} \\
u_{x}
\end{array}\right)=k \frac{\sigma^{2}}{2} N_{x}\left(\begin{array}{l}
\frac{\partial s_{x}(y)}{\partial m_{y}} \\
\frac{\partial s_{x}(y)}{\partial u_{y}}
\end{array}\right)_{y=x}
$$

14 where $x=\left(m_{x}, u_{x}\right)$ denotes a resident phenotype, and $y=\left(m_{y}, u_{y}\right)$ a mutant phenotype. $N_{x}$ is the

15 resident population size at population dynamical equilibrium, and $s_{x}(y)$ denotes the invasion fit16 ness of a mutant phenotype $y$ in a resident population of phenotype $x$. The selection gradient 17 (bracketed term on right-hand side) is a vector determining the expected local direction of the 18 adaptive process. Equation (1) extends the classical description of traits dynamics along fixed 19 adaptive landscapes to models in which the eco-evolutionary feedback between individual traits and the selective environment is made explicit (Abrams 2001; Dieckmann and Law 1996). 
The equilibria of equation (1) are the phenotypes for which both components of the selec-

2 tion gradient vanish, and are called evolutionarily singular phenotypes (Metz et al. 1992). A full

3 stability analysis of these singularities requires examining independently their evolutionary at-

4 tractivity or convergence stability, and their non-invasibility or evolutionary stability (Eshel

5 1983; Geritz et al. 1998). Local evolutionary attractivity of a singularity $\left(m^{*}, u^{*}\right)$ means that tra-

6 jectories starting in its vicinity converge to the singularity. This is guaranteed when the

7 eigenvalues of the Jacobian matrix of equation (1) have negative real parts. The local non-

8 invasibility of a singularity means that all mutants in its vicinity are unable to invade. This is

9 guaranteed when the eigenvalues of the Hessian matrix of invasion fitness (containing the second

10 derivatives with respect to the mutant phenotype) are negative (Marrow et al. 1996). Conver-

11 gence and evolutionary stability can also be characterized globally, respectively, through plotting

12 phase portraits of adaptive trajectories, and through pairwise invasibility plots (showing the sign

13 of invasion fitness $s_{x}(y)$ as a function of $x$ and $y$, Geritz et al. 1998).

\section{Spatial invasion fitness}

15 The invasion fitness of a mutant is defined as the per capita growth rate of its population when

16 rare in the environment set by the resident population (Metz et al. 1992). In Appendix A, we pre-

17 sent the construction of the population dynamics model for a single phenotype $(x)$ inhabiting the

18 network; then we extend the model to describe the interaction between $x$ and a mutant phenotype,

$19 y$ (see also Ferrière and Le Galliard 2001, and Le Galliard et al. 2003). Since all density depend-

20 ence occurs between neighboring sites, the mutant's growth over the network depends upon the

21 expected frequencies $q_{x \mid y}$ and $q_{y \mid y}$ of sites occupied respectively by a resident $(x)$ and a mutant 
1 (y) in the neighborhood of any focal mutant (Matsuda et al. 1992). Accordingly, the deterministic

2 dynamics of mutant population size $N_{y}$ are given by

$$
\frac{d N_{y}}{d t}=\left[\left(b+(1-\phi) u_{x} q_{x \mid y}+(1-\phi) u_{y} q_{y \mid y}-C\left(m_{y}, u_{y}\right)\right) q_{0 \mid y}-d\right] N_{y}
$$

6 where $\varphi=1 / n$ is the probability to draw any one of the connections at random within a given

7 neighborhood (see eq. [A2C] in Appendix A). The invasion fitness $s_{x}(y)$ is then given by the term 8 in square brackets.

Equation (2) can be understood as follows. The per capita growth rate of mutants (square 10 brackets) is obtained as the difference between their birth rate and their death rate $d$. To deter11 mine the former, the mutant's effective birth rate (large parentheses) is discounted by the 12 frequency $q_{0 \mid y}$ at which mutants find empty sites in their neighborhood. The effective birth rate is

13 given by the intrinsic birth rate $b$, enhanced by the benefits of altruism, and diminished by the 14 mutant's cost $C\left(m_{y}, u_{y}\right)$ of mobility and altruism. The benefits of altruism derive from interac15 tions between mutants and residents, $(1-\phi) u_{x} q_{x \mid y}$, and between mutants and mutants, $16(1-\phi) u_{y} q_{y \mid y}$. In both cases, the altruism rates $u_{x}$ and $u_{y}$ of the mutant's neighbors are 17 weighted by the frequencies $q_{x \mid y}$ and $q_{y \mid y}$ at which these neighbors occur in the mutant's 18 neighborhood. The factor $1-\phi$ accounts for the fact that empty and occupied sites surrounding 19 the mutant cannot be chosen independently, since their numbers must sum to $n$.

20 Even though the invasion fitness in equation (2) depends only on probabilities of finding 21 sites neighboring a mutant in certain states, the dynamics of these neighbors in turn depend on 
1 their neighbors. Therefore, a complete description of the mutant's population dynamics - and

2 thus of the probabilities $q_{x \mid y}, q_{y \mid y}$, and $q_{0 \mid y}$ in equation (2) - requires an infinite hierarchy of cor-

3 relation equations, each one describing the spatial structure at a particular scale in relation to that

4 at the next-larger one (Dieckmann et al. 2000). Here, we use the method of pair approximation to

5 close this exact system of equations at the scale of pairs (Matsuda et al. 1992; Morris 1997; see

6 Appendix A). This method is accurate for random networks featuring randomly assigned connec-

7 tions between sites, as is assumed here. Regular habitat geometry, where interactions take place

8 among the geographically closest sites, would compromise the use of the standard pair approxi-

9 mation and require more refined approximations (Ellner et al. 1998; Harada and Iwasa 1994; van

10 Baalen 2000). However, a limited set of selected individual-based simulations indicates that our

11 qualitative findings obtained from a random network model extend to regular networks when

12 using a regular square lattice (results not shown).

13 The initial population dynamics of a rare mutant involve two distinct phases (van Baalen

14 2000). First, a single mutant individual either dies without leaving any descendants, or begins to

15 invade locally until its neighborhood structure stabilizes at a pseudo-equilibrium state character-

16 ized by $\widetilde{q}_{x \mid y}, \widetilde{q}_{y \mid y}$, and $\widetilde{q}_{0 \mid y}$. Conditional on non-extinction during this first phase, the mutant

17 population expands or contracts while retaining its pseudo-equilibrium correlation structure. Spa-

18 tial invasion fitness is then calculated as the mutant population's growth rate during the second

19 stage, thus ignoring the first stage dominated by drift (van Baalen and Rand 1998),

$$
s_{x}(y)=\left(b \dashv(1-\phi) u_{x} \widetilde{q}_{x \mid y} \dashv(1-\varphi) u_{y} \widetilde{q}_{0 \mid y}-C\left(m_{y}, u_{y}\right)\right) \widetilde{q}_{0 \mid y}-d .
$$


1 A positive fitness implies that the invasion process enters a third phase during which a mutant

2 phenotype that is sufficiently similar to the resident generically displaces the resident (Geritz et 3 al. 1998).

The pseudo-equilibrium correlation structure of the mutant population is affected by the equilibrium correlation structure of the resident population, characterized by $\bar{q}_{x \mid x}$ and $\bar{q}_{x \mid 0}$. The

6 latter is obtained from a model of the monomorphic resident population (Appendix A), while the

7 former can be derived from the dynamics of a dimorphic population, when the mutant phenotype

8 is rare and the resident phenotype is at equilibrium (Appendix B). The spatial statistics $\bar{q}_{x \mid x}$ and

$9 \bar{q}_{x \mid 0}$ refine the empirical notion of habitat saturation, as originally introduced by (Brown 1978;

10 Emlen 1982). The probability $\bar{q}_{x \mid x}$ measures 'local aggregation', i.e., the level of crowding felt

11 locally by any given individual. By contrast, the probability $\bar{q}_{x \mid 0}$ measures 'local contention', i.e.,

12 the level of crowding around any vacant site, in which each neighbor might lay an offspring.

13 Since the crowding around individuals can markedly differ from the crowding around empty sites

14 it is important to strictly distinguish between these two complementary dimensions of habitat 15 saturation.

17 An analysis of the components of the selection gradient, as defined by equations (1) and (3), 18 yields a full description of the selective pressures operating on altruism and mobility. This analy19 sis is expounded in Appendix B and reveals important general insights, which we describe next. The first component of the selection gradient is the derivative of the spatial invasion fitness

21 with respect to $m$ and measures the total selective pressure on the mobility rate $m$, 


$$
\partial_{m} s_{x}(y)=\bar{q}_{0 \mid x} \frac{d}{\bar{q}_{0 \mid x}^{2}}-(1-\phi) u_{x} \partial_{m} \widetilde{q}_{0 \mid y}-\partial_{m} C
$$

4 where $\partial_{m}$ denotes a derivative with respect to $m_{y}$ evaluated at $y=x$ (see eq. [B2] in Appendix B).

5 The equation's right-hand side identifies the two competing components of this selective pres-

6 sure: the marginal physiological cost of mobility, $\partial_{m} C$ (here $\partial_{m} C=v$ ); and the marginal gain (or

7 loss) in open space resulting from altered mobility in the neighborhood of a mutant, $\partial_{m} \widetilde{q}_{0 \mid y}$,

8 translated into a fitness change via a conversion coefficient (large parentheses) depending on

9 death rate, habitat connectivity, altruism rate, and local aggregation $\bar{q}_{x \mid x}=1-\bar{q}_{0 \mid x}$. This conver-

10 sion coefficient decreases with decreasing local aggregation $\bar{q}_{x \mid x}$. Accordingly, the evolution of

11 mobility is partially driven by the advantage conferred during invasion to mutants that are sur-

12 rounded by more open space than residents. The marginal gain in open space $\partial_{m} \widetilde{q}_{0 \mid y}$ is a complex

13 function of resident mobility and local contention; numerical analysis shows that it is little influ-

14 enced by local aggregation (Appendix B).

15 The second component of the selection gradient is the derivative of spatial invasion fitness

16 with respect to $u$, which measures the total selective pressure on the altruism rate $u$ (see eq. [B1]

17 in Appendix B). Extensive numerical simulations show that the marginal gain (or loss) in open

18 space resulting from altered altruism in the neighborhood of a mutant can be neglected (see Fig-

19 ure 4 in Le Galliard et al. 2003), which yields

20

21

$$
\left.\partial_{u} s_{x}(y)=\bar{q}_{0 \mid x} \mid(1-\phi) \bar{q}_{y \mid y}-\partial_{u} C\right\rfloor
$$


2 The equation's right-hand side highlights the two competing components of this selective pres-

3 sure: the marginal, physiological cost of altruism, $\partial_{u} C$; and the benefit of increased altruism

4 among mutants, measured by $\bar{q}_{y \mid y}$ diminished by the discounting factor $1-\phi$, which solely de-

5 pends on habitat connectivity $n$. The term $\bar{q}_{y \mid y}$ measures the probability that the recipient of an

6 action performed by a mutant individual is a mutant itself, and thus provides a measure of relat-

7 edness (Day and Taylor 1998). Consequently, equation (5) emphasizes the role of kin selection in

8 the evolution of altruism. In Appendix B, relatedness $\bar{q}_{y \mid y}$ is derived analytically:

9

10

$$
\bar{q}_{y \mid y}=\frac{d \phi}{d+(1-\phi) m \bar{q}_{0 \mid x}}
$$

12 This expression shows that relatedness among mutants, and thus the marginal gain resulting from

13 altruistic interactions between relatives, is higher in populations with an elevated local aggrega-

14 tion $\bar{q}_{x \mid x}=1-\bar{q}_{0 \mid x}$ of the residents. Equation (6) also shows that the benefits of altruism rise with

15 reduced mobility, lower habitat connectivity, or reduced mortality.

16 These results demonstrate that, besides the physiological costs associated with each trait,

17 the evolutionary dynamics of altruism and mobility are mediated by two factors: eco-

18 evolutionary feedback loops and selective interactions (fig. 1). Eco-evolutionary feedback loops

19 result from the interplay between ecological variables and adaptive traits: in particular, such

20 loops occur when the change in a trait value affects the population's spatial structure, which in

21 turn modifies the selective pressures on that trait. Selective interactions in our model result from

22 the interplay between altruism and mobility. Figure 1 offers a comprehensive overview of all eco- 
1 evolutionary feedbacks and selective interactions we have identified in the joint evolution of al-

2 truism and mobility. In this map, habitat saturation, specified both in terms of local aggregation

3 and local contention, plays a central role.

Feedback between habitat saturation and mobility. Habitat saturation is entangled with mobility through a negative feedback loop that operates along two separate pathways. First, according to our exhaustive numerical tests (Appendix B), increased mobility reduces the marginal gain of open space in equation (4). Second, increased mobility decreases local aggregation

8 (through its costs on birth rate, Appendix A), which reduces the conversion coefficient in equa9 tion (4). Thus, effects along the two pathways identified here are acting synergistically to reduce 10 the selective benefits of mobility as mobility increases in the population.

11 Feedback between habitat saturation and altruism. Understanding this second feedback 12 loop starts out from the fact that altruism generally increases local aggregation (through its posi13 tive effect on birth rates, Appendix A). Local aggregation, in turn, increases relatedness and, 14 therefore, the benefits of kin cooperation described in equation (6), which favors the evolution of 15 even more altruism according to equation (5). This results in positive feedback between local 16 aggregation and altruism. In addition to the eco-evolutionary feedback, the evolution of altruism 17 is also controlled by a physiological feedback (not represented in fig. 1) whenever the cost of 18 altruism is nonlinear: for a decelerating (accelerating) cost, the marginal cost of altruism de19 creases (increases) with the level of altruism. This physiological feedback is thus positive for 20 decelerating costs of altruism and negative for accelerating costs.

21 Selective interactions between altruism and mobility. Figure 1 also allows deciphering the 22 selective interactions between altruism and mobility, in which local aggregation is crucial. On the 23 one hand, increasing mobility reduces local aggregation, which lowers relatedness according to 24 equation (6), and therefore weakens the selective pressure in favor of altruism according to equa- 
1 tion (5). On the other hand, increasing altruism strengthens local aggregation, which increases the

2 conversion factor in equation (4) and therefore the selective pressure in favor of mobility: open-

3 ing space by moving around is more beneficial when local aggregation is high. However,

4 equation (4) shows that increasing altruism also has a direct, negative effect on this conversion

5 coefficient: there is an increasing "benefit of philopatry" when neighbors are altruistic (Stacey

6 and Ligon 1991). The net effect on the evolution of mobility thus depends on the balance be-

7 tween these two counteracting effects, which varies with the level of altruism: our numerical

8 simulations reveal a net effect where the conversion coefficient is generically weakened for low-

9 est rates of altruism, and enhanced for intermediate and high altruism. Moreover, in species with

10 accelerating costs, the physiological feedback described above implies that a rise of altruism se-

11 verely diminishes fecundity, which results in reduced local aggregation, a diminished conversion

12 coefficient, hence a negative effect on the intensity of selection for opening space.

\section{SEPARATE EVOLUTION OF ALTRUISM AND MOBILITY}

14 In general, the evolutionary dynamics of single traits are monotonous and converge to a point

15 attractor (which, under certain circumstances, depends on the population ancestral state). Any

16 small mutation arising around these singularities is selected against and fails to invade.

\section{Altruism}

18 The evolutionary dynamics of altruism primarily depends upon the pattern of physiological cost

19 of altruism (see Le Galliard et al. (2003) for more details). Under the assumption of decelerating

20 costs, ancestral selfishness can only be displaced as a result of rare, large mutations. There is a

21 'waiting time' for the adaptive rise of altruism that increases with the mobility rate. Only in the

22 limiting case of a linear cost of altruism may pure selfishness remain unbeatable. This occurs in 
1 species with a 'strong' linear cost, for which $\kappa>\phi(1-\phi)$, and a mobility rate larger than the

2 threshold (see eq. [8] in Le Galliard et al. 2003)

$$
m_{l}=b \frac{\varphi(1-\varphi)-\kappa}{v(\phi(1-\phi)-\kappa)+\kappa(1-\phi)}
$$

6 Thus, the evolution of altruism is hindered only when the most unfavorable conditions are en-

7 forced, combining a high cost sensitivity to altruism, high mobility, and high habitat connectivity. In species with accelerating costs of altruism, the altruism rate evolving is lower in more

9 mobile organisms. Under 'rapidly' accelerating costs (high $\kappa$ and/or $\gamma$ much larger than 1), the 10 relationship between mobility and selected altruism is smooth, and the selected rate of altruism is

11 always low. In contrast, under 'slowly' accelerating costs (low $\kappa$ and $\gamma$ close to 1$)$ the relationship 12 between mobility and selected altruism shows a sharp discontinuity: high levels of altruism 13 evolve in species with low mobility, whereas quasi-selfishness evolves at high mobility. When 14 mobility is low, the high level of altruism that evolves can be approximated as $15 u^{*}=[\phi(1-\phi) / \kappa \gamma]^{1 /(\gamma-1)}$ (see eq. [9] in Le Galliard et al. 2003), which depends only on habitat 16 connectivity and the parameters affecting the physiological cost of altruism. At intermediate mo17 bility, the evolutionary outcome depends on the ancestral state of the population: if the ancestral 18 altruism is low, quasi-selfishness evolves; otherwise, a high level of altruism is selected.

\section{Mobility}

20 Mobility as a single adaptive trait always evolves toward a globally attractive and uninvadable

21 singularity (figs. 2A, B). In general, there is no analytical expression for the resulting ES mobility 
1 rate $m^{*}$, yet in the special case of a purely selfish species $(u=0)$, solving for the zeros of the first-

2 order Taylor expansion of spatial invasion fitness with respect to $m$ (see eq. [4]) yields

$$
m^{*}=\frac{b(\sqrt{v(1-v) \phi(1-\phi)}-v(1-v))}{v(1-v)((1-\phi)-v)} .
$$

6 Thus, in purely selfish species, the ES mobility rate decreases with an increasing cost of mobility $7 v$, and equals zero when $v \geq \varphi$ (fig. 2C); it also decreases with increasing habitat connectivity ( $n$ $8=1 / \phi$, fig. $2 \mathrm{C}$ ) and increases with the birth rate, but is independent of the mortality rate. Further9 more, the value $m^{*}$ given by eq. (8) possesses the remarkable property of maximizing the local 10 contention $\bar{q}_{x \mid 0}$; thus, in purely selfish species, evolution of mobility alone maximizes habitat 11 saturation around empty sites. Numerical simulations suggest that the same patterns persist at any 12 level of altruism $u>0$, except that higher mortality then results in a lower ES mobility rate.

13 The ES mobility rate $m^{*}$ varies also with the species' degree of altruism $u$. The empirical 14 expectation is that more altruistic species are less mobile, but the typical pattern is more complex. 15 Zero mobility is selected for if the mobility cost is too high $(v>\phi)$, irrespective of the degree of 16 altruism. Otherwise, there may be a slight decrease of $m^{*}$ as $u$ increases through very small val17 ues, but $m^{*}$ increases with $u$ over a wide range of degrees of altruism (see fig. $3 \mathrm{~A}$ for the case of 18 a decelerating altruism cost, fig. 4 for a linear cost, and figs. $2 \mathrm{~A}$ and 5 for accelerating costs). At 19 very high values of $u, m^{*}$ can decrease again with larger values of $u$ in species with accelerating costs of altruism.

This pattern can be understood from the selective pressures that operate on $m$ (see eq. [4] 
1 on the intensity of the selective pressure to open space. Furthermore, local aggregation itself de-

2 pends on the altruism rate. At extremely low values of $m$, the dependency of local aggregation on

$3 u$ is weak. Therefore, as $u$ increases, its direct, negative effect predominates, and $m^{*}$ tends to de-

4 crease. Over a range of larger $u$, local aggregation rises rapidly with $u$, so that the indirect effect

5 of $u$ via local aggregation dominates: more mobility is selected for. A further increase of $u$ causes

6 a substantial reduction in birth rate for an accelerating cost of altruism, hence a decrease of local

7 aggregation; this drives the evolution of less mobility.

9 Our analysis of the joint evolutionary dynamics of mobility and altruism develops from equation

10 (1). The two corresponding isoclines generally cross at a single attractive and evolutionarily sta-

11 ble singularity (ESS), denoted by $\left(m^{*}, u^{*}\right)$. The main conclusions of our analysis are tested 12 against numerical simulations of an individual-based model in which all approximations involved

13 in the deterministic model (1) are avoided.

\section{Origin of altruism}

15 To investigate the origin of altruism, we assume a decelerating cost of altruism. Our previous 16 analysis showed that in species characterized by such costs the conditions under which altruism 17 can evolve are the most stringent (Le Galliard et al. 2003). Also, in agreement with the classical 18 empirical view, we assume that the selfish, ancestral state involves highly mobile individuals.

19 Starting from selfishness associated with high mobility, mobility first decreases toward the criti20 cal value $m^{*}$ given by equation (8) (see figs. $\left.3 \mathrm{~A}, \mathrm{~B}\right)$. The trait pair $\left(m^{*}, 0\right)$ is an endpoint of the 21 deterministic dynamics in trait space. However, a different pattern applies when the stochasticity 22 of the underlying individual-based process is taken into account. In a population where mutations 
1 may be large occasionally, mutants characterized by a significant degree of altruism will eventu-

2 ally arise by chance, and displace the selfish resident (figs. 3C, D). Therefore, the evolutionary

3 trajectory will sooner or later take off from $\left(m^{*}, 0\right)$. It can be seen numerically that the minimum

4 value of mutant altruism required for invading the selfish resident increases as $m^{*}$ increases.

5 Thus, according to equation (8), the waiting time for altruism to evolve is shorter as the cost of

6 mobility or habitat connectivity increases, or in species with a smaller birth rate (figs. 3C, D).

Only in the case of a linear pattern of altruism cost may selfishness be always uninvadable

8 (fig. 4A), provided that the altruism cost parameter $\kappa$ is large (fig. 4B), or that both $\kappa$ and the mo-

9 bility cost $v$ are small (figs. 4C, 4D). Under such conditions, altruism may initially rise through

10 small mutational steps, if the ancestral state is not too mobile. Yet at some point in the popula-

11 tion's evolutionary history the trajectory of altruism reverts and eventually heads back to the

12 selfish state, homing in at the mobility ESS $m^{*}$ where no mutant can invade (results not shown).

13 In contrast, if $\kappa$ is small and $v$ is large enough, selfishness is readily displaced by altruism even

14 through infinitesimal mutations, as a result of selection for lower mobility (figs. 4E, F). Thus, the

15 cost of mobility has a major impact on the origin of altruism, either determining whether the dis-

16 placement of selfishness is possible (linear costs of altruism), or affecting the timescale over

17 altruism evolves (decelerating costs of altruism).

Evolutionary trajectories of social traits

19 Once the evolutionary rise of altruism from a selfish and highly mobile ancestor is initiated, the

20 assumption of an accelerating cost of altruism becomes more realistic (Le Galliard et al. 2003).

21 Then all possible evolutionary dynamics unfold along a continuum bounded by two archetypal 22 templates, each involving two distinctive evolutionary phases. 
One evolutionary template applies to species with a slowly accelerating cost of altruism

2 (low $\kappa$ and $\gamma$ close to 1 ). This template involves a first evolutionary phase characterized by the

3 evolutionary reduction of mobility, while altruism shows little change; at the same time, local

4 aggregation is enhanced (figs. 5A, B, C). During the second phase, altruism rises along with

5 some increase in mobility (fig. 5C). How this second phase ends depends on the cost of mobility.

6 In the case of a high cost of mobility, the evolutionary trajectory simply heads to the ESS (which

7 is a stable-node equilibrium). In the case of a moderate cost of mobility, the eco-evolutionary

8 feedback causes damped oscillations of the adaptive traits around the ESS (a stable-focus equilib-

9 rium, figs. 5A, B). Notice that evolution to the extinction boundary can preclude convergence to 10 the ESS (fig. 5B).

11 The other evolutionary template applies to species with rapidly accelerating costs of altru12 ism (figs. 5E, F). During the first phase of the evolutionary dynamics, the degree of altruism rises 13 while mobility and the level of local aggregation remain essentially constant. The second phase 14 drives the system to the ESS, and is characterized by a marked decrease in mobility, possibly 15 along with a further increase in altruism, while local aggregation is enhanced significantly (fig. $165 \mathrm{~F})$. In this scenario, the ES altruism rate is usually low.

\section{Evolutionarily induced correlations between altruism and mobility}

18 Physiological, life history, or environmental change can cause the ES traits to covary. The con19 ventional wisdom is that selected altruism and selected mobility should correlate negatively 20 across populations or species. Here, we analyze patterns of altruism and mobility covariation in 21 species characterized by accelerating costs of altruism, in response to underlying changes in life 22 history (birth and death rates), or in constraints on mobility (habitat connectivity and cost of mo23 bility). 
Univariate changes in life history traits $(b$ or $d$ ) lead to a negative correlation between the

2 two adaptive traits at evolutionary equilibrium: less altruism and more mobility are selected for in

3 longer-lived, or less fecund species (fig. 6A and B). However, except in species that are charac-

4 terized by low fecundity (small $b$ ) and low cost of altruism (small $\kappa$ ), the quantitative effect is

5 weak and unlikely to be amenable to empirical detection. In contrast, changes in constraints on

6 mobility ( $n$ and $v$ ) can result in an unexpected positive correlation between selected altruism and

7 selected mobility (fig. 6C and D). This correlation pattern is more pronounced for species with

8 slowly accelerating costs of altruism, and with costs of mobility spanning a range that excludes

9 extremely high and low values (fig. 6D, continuous curve).

10 These qualitative patterns can be understood by considering the selective feedbacks and in-

11 teractions governing the evolution of both adaptive traits (fig. 1). The intrinsic birth rate $b$

12 influences the evolution of mobility and altruism via an effect on local aggregation (Appendix B).

13 As $b$ increases, local aggregation increases, selecting for higher mobility and altruism. The intrin-

14 sic death rate $d$ has a direct positive effect on relatedness and a negative effect via local 15 aggregation (eq. [6]): in total, as $d$ increases, relatedness decreases, selecting for less altruism.

16 Increasing the intrinsic death rate $d$ has also a negative effect on mobility via local aggregation

17 and the conversion factor of the selective pressure for mobility (eq. [4])), thus selecting for less

18 mobility. However, as $b$ and $d$ increase, the change in mobility affects local aggregation, oppos-

19 ing the change in altruism. The negative correlation between $u^{*}$ and $m^{*}$ represents the net effect

20 of these influences acting altogether when $b$ or $d$ vary independently.

21 Variation in $n$ or $v$ may reflect different environmental constraints on individual mobility. A

22 larger value of $n$ causes a rise in the discounting factor of kin cooperation benefits (eq. [5]), and

23 reduces habitat saturation, which decreases both relatedness and the marginal gain in open space 
1 from mobility (eq. [4]). Increasing habitat connectivity $n$ therefore weakens the selective pressure

2 for both traits, and causes their joint adaptive decline. Increasing the cost of mobility $v$ over a

3 range that excludes very high and very low values promotes the evolution of significantly higher

4 altruism (for the cost of mobility is not too low), which causes a marked increase of local aggre-

5 gation. Higher local saturation in turn exerts a selective pressure for mobility, which exceeds the

6 accrued cost of mobility (for the cost of mobility is not too high). Thus, the selective interaction

7 between altruism and mobility underlies the joint rise of altruism and mobility at the ESS (fig.

8 6D).

9 DisCUSSION

10 We have used spatial invasion fitness to analyze the joint evolutionary dynamics of altruism and

11 mobility, thereby introducing a unifying framework for investigating the evolution of social

12 traits. Following on Perrin and Lehmann (2001), this allowed us to integrate two previously sepa-

13 rate lines of research that are focusing, respectively, on altruism evolution under fixed mobility

14 and on mobility evolution under fixed altruism.

15 Joint evolution versus single-trait evolution

16 Our analysis has revealed a variety of phenomena that are obscured from recognition unless altru-

17 ism and mobility are permitted to evolve jointly. First, evolutionary trajectories on higher-

18 dimensional adaptive landscape can bypass fitness valleys that are insuperable by single-trait

19 evolution. In our study this general finding applies to the repelling evolutionary isoclines of altru-

20 ism in figs. 4C, 4D, 5A, and 5B: ancestral states below these isoclines could never evolve

21 towards higher degrees of altruism, were it not for the concomitant evolution of mobility. For

22 example, when costs of altruism are linear and low, a selfish and sufficiently mobile ancestor will 
1 always be uninvadable if mobility is fixed (fig. 4C and 4D; see also Le Galliard et al. 2003). In

2 contrast, if mobility is allowed to evolve and its cost is high enough, selfishness will be readily

3 displaced, even through infinitesimal mutational steps. A similar conclusion applies in fig. 5A

4 and 5B. Joint evolution, however, may sometimes also obstruct the evolution of altruism. When

5 costs of mobility and altruism are low, and the latter is linear, altruism will increase if mobility is

6 fixed at low levels, whereas joint evolution concomitantly increases mobility, which eventually

7 drives the population back to selfishness.

The joint evolution also affects the evolution of mobility. Mobility is favored by the selec-

9 tive pressure to open space for mutants during invasion, and opposed by physiological costs. The

10 strength of the former pressure is directly and indirectly (through local aggregation) modulated

11 by the degree of altruism. More altruism weakens that selective pressure, as expected from the

12 benefits of philopatry in social species (Stacey and Ligon 1991). However, and less intuitively,

13 selection for more mobility occurs as a result of more altruism enhancing local aggregation.

14 When both traits evolve, this synergistic selective interaction between mobility and altruism af-

15 fects species with slowly accelerating costs of altruism and moderate costs of mobility (see fig.

$165 \mathrm{~A})$. In such species, mobility selected through the joint evolutionary process can be considerably

17 higher than that predicted in a selfish species, and considerably lower than that predicted in a

18 highly altruistic species. Thus, neglecting the propensity for altruism to co-evolve with mobility

19 can lead to underestimating or overestimating the level of mobility favored by natural selection.

20 The bi-dimensionality of the trait space also has a marked effect on the evolutionary dy-

21 namics of altruism when the physiological cost is slowly accelerating. In this case, the strong

22 evolutionary attractiveness of the mobility rate that maximizes fitness for intermediate degrees of

23 altruism suffices to transform evolutionary singularities that are repelling with respect to altruism

24 into attractive singularities. The joint ESS for altruism and mobility still bears the footprint of the 
1 one-dimensional instability for altruism: this ESS behaves as a focus, causing evolutionary trajec-

2 tories to spiral around it. Hence, even if populations originate in the same ancestral state and

3 share the same physiological, demographic, and ecological features, they will display high and

4 low levels of mobility and altruism (in all four possible combinations) should they be observed at

5 different epochs of their evolutionary history. This is yet another historical effect that could elu-

6 cidate comparative analyses confronting a lack of regularity in patterns of social traits and

7 potential correlates (Arnold and Owens 1999).

Correlative patterns of social traits

9 Habitat saturation models predict that, at evolutionary equilibrium, altruism and mobility should

10 correlate negatively, and more altruism, hence less mobility, should be observed in populations

11 characterized by stronger constraints on dispersal or by lower mortality (Perrin and Lehmann

12 2001). Our analysis, however, clearly shows that evolutionary outcomes cannot be predicted

13 solely from the effect of habitat saturation and its hypothesized underlying ecological or demo-

14 graphic determinants. This is because habitat saturation is a dynamic variable entangled in the

15 eco-evolutionary feedbacks involving altruism and mobility; the adaptive change of either trait

16 also has a direct effect on the selective pressure influencing the other trait; and life history traits

17 (birth and death rates) have effects on the evolutionary dynamics independently of their influence 18 on habitat saturation.

19 We find that selected altruism correlates positively with the cost of mobility and negatively

20 with habitat connectivity. We also predict a positive correlation between selected altruism and 21 mobility in response to changes in habitat connectivity or in the cost of mobility within a range 22 that excludes extremely low and high values. These results can be compared to those obtained by 23 Perrin and Lehmann (2001), who investigated the joint evolution of altruism and natal dispersal. 
1 Their kin selection model differs from ours in three crucial aspects: the habitat is structured into

2 saturated patches of given size; time is discrete and generations do not overlap; individual behav-

3 ior is influenced by kin discrimination. Irrespective of the kin discrimination mechanism, their

4 model predicts a negative correlation across populations differing in dispersal cost, and a positive

5 correlation across populations differing in patch size. The latter is a consequence of higher relat-

6 edness when patches are smaller, which favors both more altruism and more dispersal in their

7 model; exactly the same effects arise in our model when the neighborhood size shrinks. In con-

8 trast, our results depart from their finding of a negative correlation in response to increasing the

9 dispersal cost; in our model, such a negative correlation arises only for very low (and very high)

10 values of the mobility cost. This discrepancy might underline a qualitative consequence of the

11 discrimination mechanisms that Perrin and Lehmann (2001) considered (whereas altruism is un-

12 conditional in our model), which affects how altruistic benefits are distributed.

13 Our finding that low habitat connectivity or high cost of mobility selects for more altruism

14 suggests that comparative studies should find consistent relationships between physiological and

15 habitat constraints on dispersal, and levels of cooperation. Some recent intra-specific compari-

16 sons in vertebrates reported a negative effect of habitat connectivity on investment in helping

17 (e.g., Russell 2001; Spinks et al. 2000). Also, in the group of African mole rats (Bathyergidae),

18 cooperative breeding has been linked to the scarce and heterogeneous distribution of resources in

19 arid landscapes, which results in high costs of mobility (Jarvis et al. 1994). In agreement with our

20 findings, the comparative analysis of sociality (as measured by reproductive skew) yields a rough

21 correlation between costs of mobility and cooperation, with the eusocial species culminating in

22 correspondence with the most arid environment (Faulkes and Bennett 2001).

23 Empirical data relating altruism and mobility are scant, especially because quantitative as-

24 sessments of dispersal in social and asocial species are difficult to obtain. Comparative analyses 
1 of social traits in birds are still insufficient to test our prediction that more cooperation could be

2 associated with higher levels of mobility as an adaptive response to ecological constraints. How-

3 ever, the observation by (Arnold and Owens 1999) that the correlation patterns between dispersal

4 and cooperative breeding depends on the taxonomic level warrants further analyses. The occur-

5 rence of a dispersing morph in the eusocial naked mole rat Heterocephalus glaber could also be

6 the manifestation of an adaptive association between strong altruism and dispersal ability

7 (O'Riain et al. 1996). The fact that this dispersing morph participates little in cooperative activi-

8 ties further suggests that constraints on mobility might lead to a stable genetic polymorphism of

9 selfish-mobile and altruistic-sessile phenotypes, or to adaptive developmental plasticity. Al-

10 though the evolution of polymorphism was not observed in our study, it was first hypothesized by

11 van Baalen and Rand (1998), and has been reported in cellular automaton models involving regu-

12 lar lattices (Koella 2000).

13 We predict life history traits (birth and death rates) to have, in isolation, little influence on

14 the selected combination of altruism and mobility. On the one hand, an increase in the intrinsic

15 birth rate drives a decrease in altruism and an increase in mobility, although the predicted pattern

16 is fairly flat and probably difficult to detect in real data. On the other hand, more altruism evolves

17 among species with the highest mortality rates. This finding conflicts with the main conclusion of

18 Taylor and Irwin (2000) and Irwin and Taylor (2001), that altruism is more strongly favored in

19 response to lower mortality. In fact, the models by Taylor and Irwin show primarily that lower

20 mortality increases relatedness between altruists, an effect that is also found in this model. Our

21 analysis emphasizes, however, that net effect of decreasing mortality on the whole web of eco-

22 evolutionary feedback and selective interaction is to promote less altruism and more mobility.

23 On the empirical end, comparative analyses in birds have attempted to relate social behav-

24 ior with nestling mortality and adult mortality (Hatchwell and Komdeur 2000). It was found that 
1 nestling mortality had no detectable influence on the distribution of social characters (Poiani and

2 Pagel 1997), in agreement with our prediction that the intrinsic birth rate (which can be seen as 3 combining fecundity and offspring mortality) is likely to have undetectable effects. The analysis

4 of the whole available phylogeny of birds yields a pattern of stronger cooperation along with

5 lower adult mortality (Arnold and Owens 1998). This empirical pattern supports our finding of an

6 effect of the death rate, but contradicts the direction that we predict. The pattern could be recov-

7 ered in our model, however, under the assumption that lower mortality trades off across species

8 with lower fecundity, which is known to occur in birds (Arnold and Owens 1998). This suggests

9 that covariation of life history traits are important to consider when investigating the determinants

10 of patterns of social traits.

\section{The habitat saturation hypothesis}

12 Our analysis supports the view that habitat saturation is a critical nexus in the selective interac-

13 tion between altruism and mobility (Emlen 1982, 1994; Koenig et al. 1992). The 'habitat

14 saturation hypothesis' states that constraints on independent breeding favors philopatry and help-

15 ing, and provides a fruitful approach to the evolution of social traits from the empirical end. Our

16 theory leads to reexamining the basis and scope of this hypothesis, and clarifies the selective

17 pathways whereby habitat saturation influences and becomes influenced by the evolution of so18 cial traits.

19 Habitat saturation has long been regarded as a key to the evolution of social behavior. This

20 hypothesis was originally put forward to explain the evolution of cooperative breeding in birds

21 (Brown 1978; Emlen 1982), and is now underlying theories for the evolution of delayed dispersal

22 and reproductive skew (Kokko and Lundberg 2001; Reeve et al. 1998). The general view is that

23 habitat saturation drives the joint evolution of philopatry and altruism (Perrin and Lehmann 
1 2001). By offering an explicit mathematical framework to deal with the interplay of social behav-

2 ior and population dynamics, our analysis deciphers the selective pathways whereby habitat 3 saturation is involved in the evolution of social traits.

The habitat saturation hypothesis assumes that sociality evolves in two steps - the evolution of philopatry at first, next the evolution of cooperation (Helms Cahan et al. 2002). As habitat

6 saturation increases, floating and queuing before gaining access to a territory induce strong costs

7 of dispersal. This favors delayed dispersal, which would set the condition for the cost of local

8 crowding to be ameliorated by cooperating rather than simply competing (Kokko and Lundberg

9 2001). What causes habitat saturation in the first place? The scenario of 'ecological constraints'

10 asserts that environmental factors constrain mobility to low levels, hence local crowding. Such

11 environmental factors may involve habitat structure, physical predicaments to movement, or a

12 large physiological cost of moving (Jarvis et al. 1994; Russell 2001). The 'life history hypothesis'

13 assumes that habitat saturation is more likely to occur in species with low mortality, in which the

14 turnover of breeding sites would be slow (Arnold and Owens 1998).

Our analysis highlights a rather different evolutionary scenario. First, there are two distinct

16 components to habitat saturation, which play complementary roles in the evolution of social

17 traits. 'Local aggregation' $\bar{q}_{x \mid x}$ measures habitat saturation around individuals, in line with the

18 original definition of habitat saturation by Emlen (1982). 'Local contention' $\bar{q}_{x \mid 0}$ measures habi-

19 tat saturation around vacant sites; it is directly related to the degree of clustering of vacant sites

$20 \quad\left(\bar{q}_{0 \mid 0}=1-\bar{q}_{x \mid 0}\right)$, and thus measures how isolated groups of occupied sites are. Like in the ecologi-

21 cal constraints scenario, a high cost of dispersal and low habitat connectivity are important

22 determinants of the evolution of local aggregation and local contention. However, our model em-

23 phasizes that habitat saturation is a consequence of the evolution of low mobility, rather than the 
1 primary selective factor for that evolution. In other words, philopatry is an adaptive response to

2 environmental constraints and physiological costs, rather than to habitat saturation per se.

In fact, neither local aggregation nor local contention is maximized during the joint evolu-

4 tion of altruism and mobility. In single-trait evolution, however, the mobility rate evolves such as

5 to maximize local contention - a prediction qualitatively similar to the finding that the number

6 of competitors for territories (the limiting resource) is typically maximized by the evolution of

7 habitat choice strategies (Kokko et al. 2001). Yet this remarkable principle evaporates when al-

8 truism evolves concomitantly with mobility. Furthermore, the evolution of low mobility and

9 strong aggregation does not appear as an obligate evolutionary step toward sociality. The model

10 presented here uncovers an alternative scenario according to which a population initiated in the

11 selfish and highly mobile ancestral state first evolves a substantial degree of altruism, while ag-

12 gregation remains low, before adaptive evolution secondarily favors reduced mobility, which 13 may in turn lead to strong aggregation.

14 One key feature of our analysis is that habitat saturation is not treated as a fixed parameter,

15 but as a pair of dynamical variables that close the eco-evolutionary feedback loops entangling

16 altruism and mobility. Local aggregation and local contention, respectively, are the pivotal fac-

17 tors of the two eco-evolutionary feedback loops linking altruism and mobility. When both traits

18 evolve jointly, local aggregation turns out to be the dominant mediator of the selective interaction

19 between them. Local aggregation responds antagonistically to evolutionary change of altruism

20 and mobility, which in turn affects the selective pressures acting on both traits. Such essential

21 eco-evolutionary feedbacks and selective interactions have been ignored in most previous models

22 of social evolution (but see Kokko and Lundberg 2001).

23 The dynamical nature of local aggregation and contention in our model results from the

24 stochastic nature of the demographic process, and especially from the site opening process gener- 
1 ated by individual mortality. The numerical model of Mitteldorf and Wilson (2000) also showed

2 that population elasticity, i.e. variable local density, can facilitate the evolution of altruism, even

3 when generations do not overlap. In Nakamaru et al.'s $(1997,1998)$ models, the availability of

4 empty space was also a critical feature for the spread of cooperation. These authors further em-

5 phasized the consequences of assuming survival altruism rather than reproduction altruism. In the

6 latter case (that is considered here), the evolution of social traits affects the birth rate and, there-

7 fore, does not feedback on the site opening process, driven by mortality. If altruism impacts

8 survival, such a feedback could exist. The adaptive increase of altruism would reduce the death

9 rate, hence the rate of site opening: as a consequence, the selective pressure of local competition

10 against altruism would be enhanced. Such a negative effect on the evolution of altruism might be

11 offset, however, would some form of environmental stochasticity drive site opening, insensitive

12 to the evolutionary change of altruism (Mitteldorf and Wilson 2000).

\section{Concluding remarks}

14 The 'habitat saturation hypothesis', the 'ecological constraint model', and the 'life history hy-

15 pothesis' represent varied attempts at singling out general factors of social evolution. By

16 integrating some of their key ingredients, our model leads to the conclusion that no simple deter-

17 minism should be expected for the origin of social behavior or the evolution of strong cooperative

18 interaction. Thus, inferences from studies based on univariate analyses are likely to be hindered

19 by the complexity and diversity of factors involved in the evolution of social traits (Crespi and

20 Choe 1997). However, some general principles hold: physiological or ecological constraints on

21 mobility are essential to explain the origin of altruism; all evolutionary trajectories can be related

22 to only two archetypal, contrasting routes to sociality; patterns of covariation among social traits

23 can be understood as adaptive responses to multivariate changes in life history traits. 
Eco-evolutionary feedbacks and selective interactions are central to the joint evolutionary

2 dynamics of social traits. Taking them into account allowed us to address a hotly debated issue in

3 the biology of social behavior: whether the high relatedness between interacting individuals of

4 several social species predicted by Hamilton's kin selection theory $(1964 a, b)$ is the direct conse-

5 quence of physiological or ecological constraints on dispersal, or the outcome of more involved

6 mechanisms of active assortment, involving communication, cognition, and habitat choice

7 (Hamilton 1975). We offer the alternative view that in some social systems, both limited mobility

8 and strong altruism form the joint adaptive response to a web of multiple, interacting selective

9 mechanisms; while in other systems, the spatial self-structuring of population leads to the evolu-

10 tion of high mobility without compromising the likelihood of passive assortment between

11 altruistic partners.

12

13 Acknowledgements. We are grateful to Nicolas Perrin, Laurent Lehmann and one anonymous 14 referee for comments on an earlier version of this manuscript. This work has been financially 15 supported by the Adaptive Dynamics Network at the International Institute for Applied System 16 Analysis (Laxenburg, Austria); the French Ministry of Research and Education; the Austrian Sci17 ence Fund; the Austrian Federal Ministry of Education, Science, and Cultural Affairs; and the 18 European Science Foundation's Theoretical Biology of Adaptation Programme. Collaboration on 19 this study has been fostered by the European Research Training Network ModLife (Modern Life20 History Theory and its Application to the Management of Natural Resources), supported by the 21 Fifth Framework Programme of the European Community. 
2 We consider a social network comprising a large number of homogeneous sites, occupied by a 3 population of mutants, called $y$, and residents, denoted by $x$. A mutant $y$ located at a site $z$ on the 4 network experiences the following birth, death, and movement rates, 5

6

$$
b_{y}(z)=\left(b+\sum_{j=x, y} \phi u_{j} n_{j \mid y}(z)-C\left(m_{y}, u_{y}\right)\right) \phi n_{0 \mid y}(z)
$$

$$
d_{y}(z)=d
$$

$$
m_{y}(z)=m \varphi n_{0 \mid y}(z)
$$

10 To derive the dynamics of the mutant's population size, we average birth and death rates de-

11 scribed in equation (A1) over all sites of the network occupied by the mutant, which gives

$$
\frac{d N_{y}}{d t}=\left(\left(b-C\left(m_{y}, u_{y}\right)\right) \phi E\left(n_{0 \mid y}(z)\right)-d\right) N_{y}+\sum_{j=(x, y)} \phi^{2} u_{j} \sum_{z} n_{j \mid y}(z) n_{0 \mid y}(z)
$$

15 where $E\left(n_{0 \mid y}(z)\right)$ is the network average of the number of empty sites neighboring a site occu16 pied by a mutant. The third term in (A2a) is a product between random variables describing 17 alternative neighborhoods of a mutant individual. Assuming a multinomial probability distribu18 tion of sites and independence between the neighborhoods of pairs of sites (Morris 1997), we 19 have 
$1 \quad \sum_{z} n_{j \mid y}(z) n_{0 \mid y}(z)=N_{y} n(n-1) q_{j \mid y} q_{0 \mid y}$

3 where $q_{k \mid y}$ is the average local frequency of type- $k$ sites neighboring a mutant. The dynamics of

4 the mutant's population size is then given by

5

6

$$
\frac{d N_{y}}{d t}=\left(\left(b+\sum_{j=(x, y)}(1-\phi) u_{j} q_{j \mid y}-C\left(m_{y}, u_{y}\right)\right) q_{0 \mid y}-d\right) N_{y}=\lambda_{y} N_{y}
$$

7

8 which involves the configurations of pairs of sites. A closed system describing the pair dynamics

9 is obtained in Le Galliard et al. (2003) from the bookkeeping of all events affecting pairs of sites,

10

11

$$
\frac{d N_{0 y}}{d t}=\left(\alpha_{y}^{\prime} q_{0 \mid 0}-\beta_{y}-\delta_{y}\right) N_{0 y}+\delta_{x} N_{x y}+\delta_{y} N_{y y}
$$

12

$$
\frac{d N_{y y}}{d t}=2 \beta_{y} N_{0 y}-2 \delta_{y} N_{y y}
$$

13

$$
\frac{d N_{x y}}{d t}=\left(\alpha_{x}+\alpha^{\prime}{ }_{y} q_{x \mid 0}\right) N_{0 y}-\left(\delta_{x}+\delta_{y}\right) N_{x y}
$$

14

where $a_{i}$ is the average per capita input rate of a type- $i$ individual into a type- $0 j$ pair with $j \neq i$

$16\left(a_{i}=\alpha^{\prime}{ }_{i} q_{i \mid 0}\right), \beta_{i}$ is the average per capita input rate of a type- $i$ individual into a type- $0 i$ pair, and

$17 \delta_{i}$ is the average per capita output rate of a type-i individual from a type- $i j$ pair (following van

18 Baalen and Rand 1999, see also Appendix 2 in Le Galliard et al. 2003). 
In general, a resident population converges to a unique stable equilibrium spatial structure,

2 described in the Appendix 3 of Le Galliard et al. (2003). The non-trivial population equilibrium is 3 characterized by $\bar{q}_{x \mid x}$, which satisfies the quadratic equation

$4 \quad\left(\left(b+u_{x}(1-\varphi) \bar{q}_{x \mid x}-C\left(u_{x}, m_{x}\right)\right)\left(1-\bar{q}_{x \mid x}\right)-d=0\right.$, and by $\bar{q}_{0 \mid 0}=\delta_{x} / a^{\prime}{ }_{x}$. If $b$ is sufficiently larger

5 than $d$, the resident population is non-viable when $\Delta<0$, where $\Delta$ denotes the discriminant of the 6 quadratic equation.

Pseudo-equilibrium frequencies. We use the accents ' $\sim$ ' and '-' to denote the pseudo-

9 equilibrium state of the mutant during invasion and the equilibrium state of the resident, respec-

10 tively. The pseudo-equilibrium frequencies $\widetilde{q}_{0 \mid y}, \widetilde{q}_{x \mid y}$, and $\widetilde{q}_{y \mid y}$ are the steady states of equation

11 (A3) when $x$ is a resident type at ecological equilibrium and $y$ is a rare mutant type, which gives

12

13

14

15

16 Since $\widetilde{q}_{y \mid 0} \approx 0$ when the mutant is rare, this nonlinear system involves three unknowns $\left(\widetilde{q}_{0 \mid y}\right.$,

$17 \widetilde{q}_{x \mid y}$, and $\widetilde{q}_{y \mid y}$ ) and two equations. Together with the constraint $\widetilde{q}_{0 \mid y}=1-\widetilde{q}_{x \mid y}-\widetilde{q}_{y \mid y}$, equations

18 (B1) can thus be used to evaluate the pseudo-equilibrium frequencies of the mutant, and hence

19 the spatial invasion fitness defined by equation (3) in the main text. 
2 solution for the pseudo-equilibrium frequencies of a mutant. However, assuming a degenerate

3 mutant with the same phenotype as the resident, the non-linear system (B1) can be solved ana-

4 lytically. The solutions of equations (B1) in this case are $\widetilde{q}_{0 \mid y}=\bar{q}_{0 \mid x}$ and $\widetilde{q}_{y \mid y}=\bar{q}_{y \mid y}$ where the

5 detailed analytical expression for $\bar{q}_{y \mid y}$ (the relatedness in our model) is given by equation (6).

7 can be approximated by a first-order Taylor expansion of the spatial invasion fitness with respect 8 to $m$. Considering a slightly different mobility phenotype $m_{y}=m_{x} \dashv \mathcal{E}$, and the first-order ap9 proximations $\widetilde{q}_{0 \mid y}=\bar{q}_{0 \mid x} \dashv a \varepsilon$ and $\widetilde{q}_{y \mid y}=\bar{q}_{y \mid y} \dashv b \varepsilon$ leads to

$$
\left.\frac{\partial s_{x}(y)}{\partial m_{y}}\right|_{m_{y}=m_{x}}=\bar{q}_{0 \mid x}\left(\left(\frac{d}{\bar{q}_{0 \mid x}{ }^{2}}-(1-\phi) u_{x}\right) a-\left.\frac{\partial C\left(m_{y}, u_{x}\right)}{\partial m_{y}}\right|_{m_{y}=m_{x}}\right)+o(\varepsilon)
$$

13 The analytical evaluation of $a$ using equations (B1) yields a complicated term affected directly by 14 the mobility and altruism rate, death rate, cost of mobility, and neighborhood size, but also indi15 rectly by the effects of all model parameters on the habitat saturation statistics $\bar{q}_{x \mid x}$ and $\bar{q}_{0 \mid 0}$.

16 Numerical sensitivity analyses of the selection components over a large range of parameter val17 ues indicate that $a$ is primarily sensitive to changes in mobility rates through local contention $18 \bar{q}_{x \mid 0}$, with a negative feedback of $m$ on this selection component. For example, assuming a zero 19 mobility cost, local aggregation becomes independent of mobility, whereas local contention in20 creases monotonically with the mobility rate; thus, in this case, the eco-evolutionary feedback on 21 mobility is mediated entirely by local contention, and not by local aggregation. The conversion 
1 term (bracketed expression in front of $a$ ) is primarily sensitive to changes in altruism rate, life 2 history traits, and habitat structure.

Selective pressure on altruism. The second component of the selection gradient can be ap4 proximated by a first-order Taylor expansion of the spatial invasion fitness with respect to $u$. 5 Assuming a slightly deviant mutant $u_{y}=u_{x} \dashv \varepsilon, \widetilde{q}_{0 \mid y}=\bar{q}_{0 \mid x} \dashv a^{\prime} \varepsilon$ and $\widetilde{q}_{y \mid y}=\bar{q}_{y \mid y} \dashv b^{\prime} \varepsilon$, the first6 order approximation results in the following expression (see also eq. [3] in Le Galliard et al. 7 2003):

8

9

$$
\left.\frac{\partial s_{x}(y)}{\partial u_{y}}\right|_{u_{y}=u_{x}}=\bar{q}_{0 \mid x}\left((1-\phi) \bar{q}_{y \mid y}+\left(\frac{d}{\bar{q}_{0 \mid x}^{2}}-(1-\phi) u_{x}\right) a^{\prime}-\left.\frac{\partial C\left(m_{x}, u_{y}\right)}{\partial u_{y}}\right|_{u_{y}=u_{x}}\right)+o(\varepsilon) .
$$


2 Abrams, P. A. 2001. Modelling the adaptive dynamics of traits involved in inter- and intraspecific interactions: An assessment of three methods. Ecology Letters 4:166-175.

4 Arnold, K. E., and I. P. F. Owens. 1998. Cooperative breeding in birds: a comparative test of the life-history hypothesis. Proceedings of the Royal Society London B 265:739-745.

$6-$ 1999. Cooperative breeding in birds: the role of ecology. Behavioral Ecology 10:465-471.

7 Brown, J. L. 1978. Avian communal breeding systems. Annual Review of Ecology and Systemat$8 \quad$ ics 9:123-155.

9 Choe, J. C., and B. J. Crespi. 1997, The evolution of social behavior in insects and arachnids. 10 Cambridge, Cambridge University Press.

11 Clutton-Brock, T. 2002. Breeding together: kin selection and mutualism in cooperative verte12 brates. Science 296:69-72.

13 Clutton-Brock, T. H., D. Gaynor, R. Kansky, A. D. C. MacColl, G. McIlrath, P. Chadwick, P. N. 14 M. Brotherton et al. 1998. Costs of cooperative behaviour in suricates (Suricata suricatta). 15 Proceedings of the Royal Society London B 265:185-190.

16 Cockburn, A. 1998. Evolution of helping behavior in cooperatively breeding birds. Annual Re17 views of Ecology and Systematics 29:141-177.

18 Cohen, D., and U. Motro. 1989. More on optimal dispersal: taking into account the cost of dis19 persal mechanism. The American Naturalist 134:659-663.

20 Crespi, B. J. 2001. The evolution of social behavior in microorganisms. Trends in Ecology and $21 \quad$ Evolution 16:178-183. 
1 Crespi, B. J., and J. C. Choe. 1997. Explanation and evolution of social systems in J. C. Choe, and B. J. Crespi, eds. The evolution of social behavior in insects and arachnids. Cambridge, Cambridge University Press.

Day, T., and P. D. Taylor. 1998. Unifying genetic and game theoretic models of kin selection for continuous traits. Journal of Theoretical Biology 194:391-407.

Dieckmann, U., and R. Law. 1996. The dynamical theory of coevolution: a derivation from stochastic ecological processes. Journal of Mathematical Biology 34:579-612.

Dieckmann, U., R. Law, and J. A. J. Metz. 2000, The geometry of ecological interactions: simplifying spatial complexities. Cambridge, Cambridge University Press.

Ellner, S. P., A. Sasaki, Y. Haraguchi, and H. Matsuda. 1998. Speed of invasion in lattice population models:pair-edge approximation. Journal of Mathematical Biology 36:469-484.

Emlen, S. T. 1982. The evolution of helping. I. An ecological constraints model. The American Naturalist 119:29-39.

—. 1994. Benefits, constraints and the evolution of family. Trends in Ecology and Evolution 9:282-285.

- 1997. Predicting family dynamics in social vertebrates, Pages 228-253 in J. R. Krebs, and N. B. Davies, eds. Behavioural ecology: an evolutionary approach. Oxford, Blackwell Science.

Eshel, I. 1983. Evolutionary and continuous stability. Journal of Theoretical Biology 103:99-111. Faulkes, C. G., and N. C. Bennett. 2001. Family values: group dynamics and social control of reproduction in African mole-rats. Trends in Ecology and Evolution 16:184-190.

Ferrière, R., and J.-F. Le Galliard. 2001. Invasion fitness and adaptive dynamics in spatial population models, Pages 57-79 in J. Clobert, E. Danchin, A. A. Dhondt, and J. Nichols, eds. Dispersal. Oxford, Oxford University Press. 
1 Frank, S. A. 1995. Mutual policing and repression of competition in the evolution of cooperative groups. Nature 377:520-522.

—. 1998, Foundations of social evolution: Monographs in Behavior and Ecology. Princeton, Princeton University Press.

Geritz, S. A. H., E. Kisdi, G. Meszena, and J. A. J. Metz. 1998. Evolutionary singular strategies and the adaptive growth and branching of the evolutionary tree. Evolutionary Ecology $12: 35-57$

Gillespie, D. T. 1976. A general method for numerically simulating the stochastic time evolution of coupled chemical reactions. Journal of Computational Physics 22:403-434.

Grafen, A. 1984. Neutral selection, kin selection, and group selection, Pages 62-84 in J. R. Krebs, and N. B. Davies, eds. Behavioral Ecology. Oxford, Blackwell.

Hamilton, W. D. 1964a. The genetical evolution of social behaviour, I. Journal of Theoretical Biology 7:1-16.

—. 1964b. The genetical evolution of social behaviour, II. Journal of Theoretical Biology 7:1752.

—. 1975. Innate social aptitudes of man: an approach from evolutionary genetics, Pages 133-155 in R. Fox, ed. Biosocial Anthroplogy. New York, Wiley.

Harada, Y., and Y. Iwasa. 1994. Lattice population dynamics for plants with dispersing seeds and vegetative propagation. Research on Population Ecology 36:237-249.

Hatchwell, B. J., and J. Komdeur. 2000. Ecological constraints, life history traits and the evolution of cooperative breeding. Animal Behaviour 59:1079-1086.

Helms Cahan, S., D. T. Blumstein, L. Sundström, L. Liebig, and A. Griffin. 2002. Social trajectories and the evolution of social behaviour. Oikos 96:206-216. 
1 Irwin, A. J., and P. D. Taylor. 2001. Evolution of altruism in stepping-stone populations with overlapping generations. Theoretical Population Biology 60:315-325.

Jarvis, J. U. M., M. J. O'Riain, N. C. Bennett, and P. W. Sherman. 1994. Mammalian eusociality: a family affair. Trends in Ecology and Evolution 9:47-51.

Kelly, J. K. 1994. The effect of scale dependent processes on kin selection: mating and density regulation. Theoretical Population Biology 46:32-57.

Koella, J. 2000. The spread of altruism versus the evolutionary response of egoists. Proceedings

Koenig, W. D., F. A. Pitelka, W. J. Carmen, R. L. Mumme, and M. T. Stanback. 1992. The evolution of delayed dispersal in cooperative breeders. The Quaterly Review of Biology 67:111-150.

Kokko, H., and P. Lundberg. 2001. Dispersal, migration, and offspring retention in saturated habitats. The American Naturalist 157:188-202.

Kokko, H., W. J. Sutherland, and R. A. Johnstone. 2001. The logic of territory choice: Implications for conservation and source-sink dynamics. The American Naturalist 157:459-463.

Le Galliard, J.-F., R. Ferrière, and U. Dieckmann. 2003. The adaptive dynamics of altruism in spatially heterogeneous populations. Evolution 57:1-17.

Marrow, P., U. Dieckmann, and R. Law. 1996. Evolutionary dynamics of predator-prey systems: an ecological perspective. Journal of Mathematical Biology 34:556-578.

Matsuda, H., N. Ogita, A. Sasaki, and K. Sato. 1992. Statistical mechanics of population - The lattice Lotka-Volterra model. Progress of Theoretical Physics 88:1035-1049.

Metz, J. A. J., R. M. Nisbet, and S. A. H. Geritz. 1992. How should we define fitness for general ecological scenarios? Trends in Ecology and Evolution 7:198-202. 
1 Michod, R. E. 1999, Darwinian dynamics - Evolutionary transitions in fitness and individuality. Princeton, Princeton University Press.

Mitteldorf, J., and D. S. Wilson. 2000. Population viscosity and the evolution of altruism. Journal of Theoretical Biology 204:481-496.

Morris, A. 1997. Representing spatial interactions in simple ecological models. Thesis Dissertation, University of Warwick, Coventry.

Nakamaru, M., H. Matsuda, and Y. Iwasa. 1997. The evolution of cooperation in a latticestructured population. Journal of Theoretical Biology 184:65-81.

Nakamaru, M., H. Nogami, and Y. Iwasa. 1998. Score-dependent fertility model for the evolution of cooperation in a lattice. Journal of Theoretical Biology 194:101-124.

O'Riain, M. J., J. U. M. Jarvis, and C. G. Faulkes. 1996. A dispersive morph in the naked molerat. Nature 380:619-621.

Perrin, N., and L. Lehmann. 2001. Is sociality driven by the costs of dispersal or the benefits of philopatry? A role for kin-discrimination mechanisms. The American Naturalist 158:471483.

Poiani, A., and M. Pagel. 1997. Evolution of avian cooperative breeding: comparative tests of the nest predation hypothesis. Evolution 51:226-240.

Queller, D. C. 1992. Does population viscosity promote kin selection? Trends in Ecology and Evolution 7:322-324.

Rand, D. 1998. Correlation equations and pair approximation for spatial ecologies, Pages 100143 in J. Mc Glade, ed. Advanced ecological theory, Blackwell Science.

Reeve, H. K., S. T. Emlen, and L. Keller. 1998. Reproductive sharing in animal societies: reproductive incentives or incomplete control by dominant breeders ? Behavioral Ecology 9:267278. 
1 Russell, A. F. 2001. Dispersal costs set the scene for helping in an atypical avian cooperative breeder. Proceedings of the Royal Society London B 268:95-99.

Spinks, A. C., J. U. M. Jarvis, and N. C. Bennett. 2000. Comparative patterns of philopatry and dispersal in two common mole-rat populations: implications for the evolution of mole-rat sociality. Journal of Animal Ecology 69:224-234.

Stacey, P. B., and J. D. Ligon. 1991. The benefits-of-philopatry hypothesis for the evolution of cooperative breeding : variation in territory quality and group size effects. The American Naturalist 137:831-846.

Taylor, P. D. 1992. Inclusive fitness in a homogeneous environment. Proceedings of the Royal Society London B 249:299-302.

Thorne, B. L. 1997. Evolution of eusociality in termites. Annual Review of Ecology and Systematics 28:27-54.

van Baalen, M. 2000. Pair approximation for ecological interactions on different geometries, Pages 359-387 in U. Dieckmann, R. Law, and J. A. J. Metz, eds. The geometry of ecological interactions: simplifying spatial complexities. Cambridge, Cambridge University Press. van Baalen, M., and D. Rand. 1998. The unit of selection in viscous populations and the evolution of altruism. Journal of Theoretical Biology 193:631-648.

West, S. A., M. G. Murray, C. A. Machado, A. S. Griffin, and E. A. Herre. 2001. Testing Hamilton's rule with competition between relatives. Nature 409:510-513.

Wilson, D. S., G. B. Pollock, and L. A. Dugatkin. 1992. Can altruism evolve in purely viscous populations? Evolutionary Ecology 6:331-341. 
Table 1. Notations used in this paper.

\begin{tabular}{|c|c|}
\hline \multicolumn{2}{|c|}{ Model parameters } \\
\hline$n$ & Neighborhood size (habitat connectivity) \\
\hline$\phi=1 / n$ & Probability to draw a connection at random within a given neighborhood \\
\hline$b$ & Intrinsic per capita birth rate \\
\hline$d$ & Intrinsic per capita death rate \\
\hline$m$ & Intrinsic per capita mobility rate (adaptive trait) \\
\hline$u$ & Intrinsic per capita rate of investment in altruism, or altruism rate (adaptive trait) \\
\hline$C(m, u)$ & Cost of mobility and altruism diminishing the birth rate \\
\hline$\kappa$ & Cost sensitivity with respect to the altruism rate \\
\hline$\gamma$ & Cost acceleration with respect to the altruism rate \\
\hline$v$ & Cost sensitivity with respect to the mobility rate \\
\hline$k$ & Mutation probability per birth event \\
\hline$\sigma^{2}$ & Mutational step variance \\
\hline \multicolumn{2}{|c|}{ Model variables } \\
\hline$n_{k \mid i}(z)$ & Number of sites in state $k$ neighboring a site in state $i$ at location $z$ \\
\hline$n_{k \mid i j}(z)$ & Number of sites in state $k$ neighboring a site in state $i$ within a pair $i j$ at location $z$ \\
\hline$N_{i}$ & Number of sites in state $i$ \\
\hline$N_{i j}$ & Number of pairs in state $i j$ \\
\hline$q_{i \mid j}$ & Average local frequency of sites in state $i$ neighboring a site in state $j$ \\
\hline$q_{i \mid j k}$ & Average local frequency of sites in state $i$ neighboring a site in state $j$ within a pair $j k$ \\
\hline
\end{tabular}

Note: Subscript $x$ and $y$ refer to a resident and a mutant phenotype, respectively. 
2 Figure 1. Selective pathways affecting altruism and mobility. All eco-evolutionary feedbacks and 3 selective interactions can be traced on this diagram. Curved gray arrows indicate selective pres4 sures (see text for explanations). Plain arrows refer to positive or negative links established from 5 the analysis of equations (4) to (6) (see also Appendix B). The dotted arrow indicates a complex

6 combination of direct and indirect (via local contention) effects of mobility on the marginal gain

7 in open space. * Relatedness and the marginal gain in open space are measured for a mutant dur8 ing invasion.

9 Figure 2. Evolutionary dynamics of mobility. A, Singular mobility rates for an accelerating cost 10 of altruism. Gray curve: mobility isocline. Arrows: selection gradients. Dashed curves: contour 11 lines of the local contention $\bar{q}_{x \mid 0}$. Dark area: population extinction domain. Parameter values: $\gamma=$

$123, \kappa=0.001$ and $v=0.1$. B, Average of ten independent stochastic simulations (continuous 13 curves) and deterministic approximation (dashed curves) at $u_{x}=0$ (two lower curves), $u_{x}=20$ 14 (two intermediate curves), or $u_{x}=10$ (two upper curves). Mutation parameters: $k=0.01$ and $\sigma=$ 15 0.01. C, Evolutionarily stable mobility rates with respect to the cost of mobility, for different val16 ues of habitat connectivity. Other parameters values as in A.

17 Figure 3. Joint evolution of altruism and mobility with decelerating costs of altruism. A, Bistabil18 ity in the evolutionary dynamics of altruism. For any mobility rate, the population evolves toward 19 selfishness if initial altruism is below a threshold; above that threshold, higher altruism evolves. 20 Continuous gray curve: attractive mobility isocline. Continuous black curve: attractive altruism 21 isocline. Dashed black curve: repelling altruism isocline. Arrows: selection gradients. Open cir- 
1 cle: repelling singularity. Filled circle: attractive singularity. Parameters values: $\gamma=0.5, \kappa=0.2$,

2 and $v=0.05$. B, Average of ten stochastic simulations at four different initial conditions (con-

3 tinuous curve) and the deterministic predictions (dashed curves). Mutation parameters: $k=0.01$,

$4 \sigma=0.01$. C, Threshold altruism rate for a mutant to invade a selfish resident at the ES mobility

5 rate. Effect of the cost of mobility for different values of the cost acceleration. The limit case of a

6 linear cost is indicated by a vertical dashed line.

7 Figure 4. Joint evolution of altruism and mobility with a linear cost of altruism. A, Convergence 8 to selfishness under low cost sensitivity to altruism, $\kappa<\phi(1-\phi)$, and low costs of mobility. Pa-

9 rameter values: $\kappa=0.15$ and $v=0.01$. B, Stochastic trajectories. Average of ten stochastic 10 simulations (continuous curve), and deterministic approximation (dashed curves). Parameter val-

11 ues as in A. C, Divergence to more altruism under low cost sensitivity to altruism, and high costs 12 of mobility. Parameter values: $\kappa=0.1$ and $v=0.1$. D, Stochastic trajectories. Parameter values as 13 in C. Filled circles indicate attractive ESSs. Arrows give the direction of evolution. Mutation 14 parameters: $k=0.01$ and $\sigma=0.05$ in B and $\sigma=0.01$ in D. Other parameter values as in fig. 2 .

15 Figure 5. Joint evolution of altruism and mobility with an accelerating cost of altruism. A, Con16 vergence to a stable focus under slowly accelerating costs of altruism and intermediate costs of 17 mobility. Parameter values: $\gamma=1.2, \kappa=0.05$, and $v=0.05$. B, Stochastic trajectories. Trajectories 18 differ quantitatively from the deterministic approximation, but remain qualitatively similar in this 19 case. Finite population size and random mutational steps induce contingency: starting from the 20 same mobile, altruistic ancestor, trajectories can either converge to the focus or collide with the 21 extinction boundary (triangle). C, Relationship between local aggregation and mobility (gray 22 curve) or altruism (black curve) in the course of adaptive evolution (arrows) from stochastic tra- 
1 jectories. Parameter values as in A. D, Convergence to a stable node under rapidly accelerating

2 costs of altruism. Parameter values: $\gamma=2, \kappa=0.05$, and $v=0.1$. E, Stochastic trajectories. Pa-

3 rameter values as in D. F, Relationship between local aggregation and mobility (gray curve) or

4 altruism (black curve) in the course of adaptive evolution (arrows). Parameter values as in D,

5 except $\kappa=0.5$. Mutation parameters: $k=0.01$ and $\sigma=0.01$. Filled circles indicate attractive

6 ESSs. Arrows show the selection gradients. Dark areas indicate population extinction. Other pa-

7 rameter values as in fig. 2.

8 Figure 6. Correlations between altruism and mobility induced by evolution with accelerating 9 costs of altruism. Arrows indicate the effect of increasing the parameter value. A, Effect of vary10 ing the birth rate, $b$, for different values of the cost parameter $\kappa$. Parameter values: $\gamma=2$ and $v=$

11 0.1. B, Effect of varying the death rate, $d$, for different values of $\kappa$. Parameters: $\gamma=2, v=0.1$, and $12 b=6$. In A and $\mathrm{B}, \kappa=0.01, \kappa=0.02, \kappa=0.05, \kappa=0.1$ from top to bottom. C, Effect of habitat 13 connectivity, $n$, for different values of $\kappa$. Parameter values: $\gamma=2.5$ and $v=0.1$. From top to bot14 tom: $\kappa=0.005, \kappa=0.01, \kappa=0.05, \kappa=0.1$. D, Effect of the cost of mobility, $v$, for different 15 levels of cost acceleration: $\gamma=1.2$ (continuous curve, results from stochastic simulations), $\gamma=1.5$ 16 (dashed curve), $\gamma=2$ (dotted curve). Parameter values: $\kappa=0.05$. Other parameters as in fig. 2 . 
Figure 1

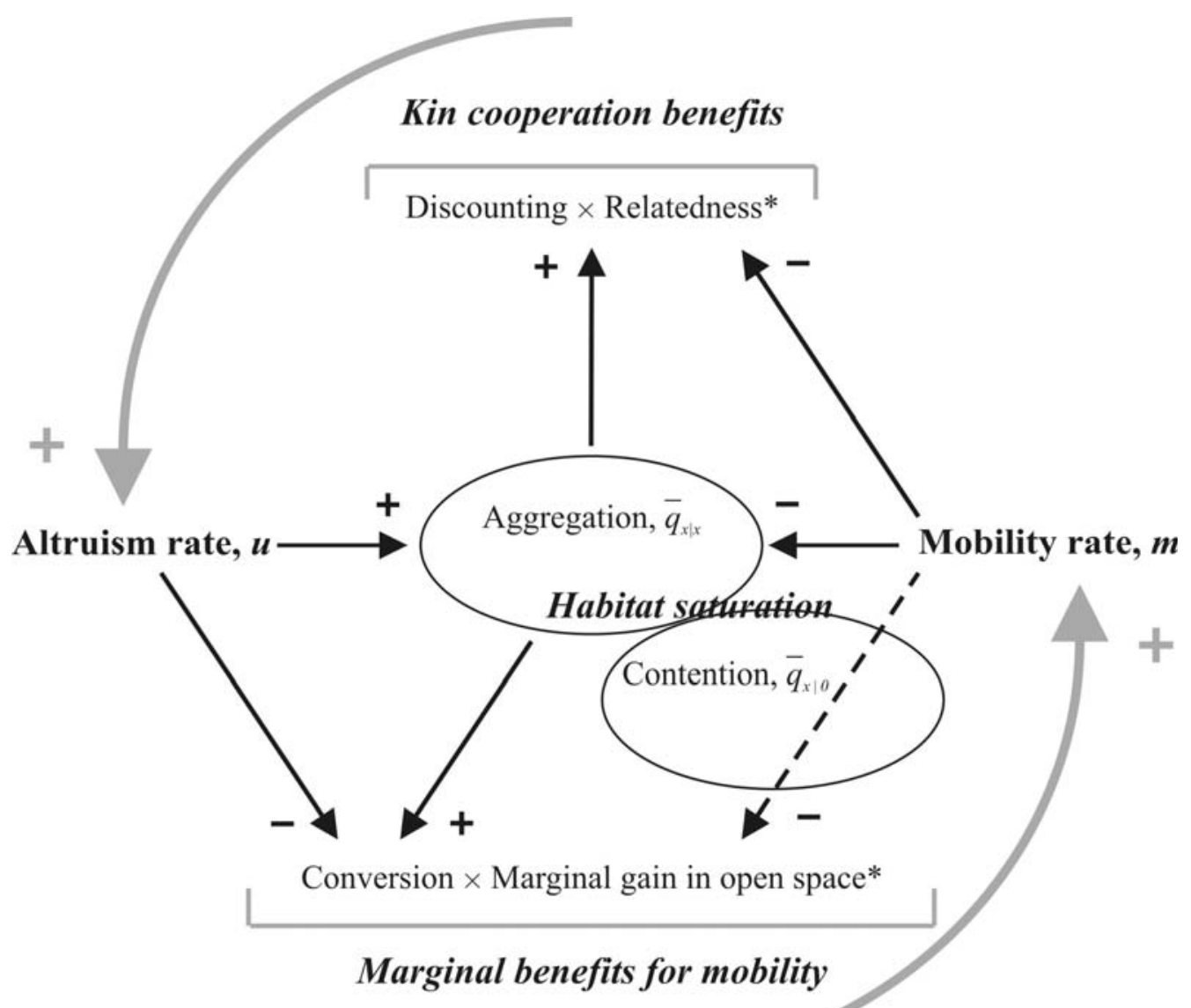


Figure 2
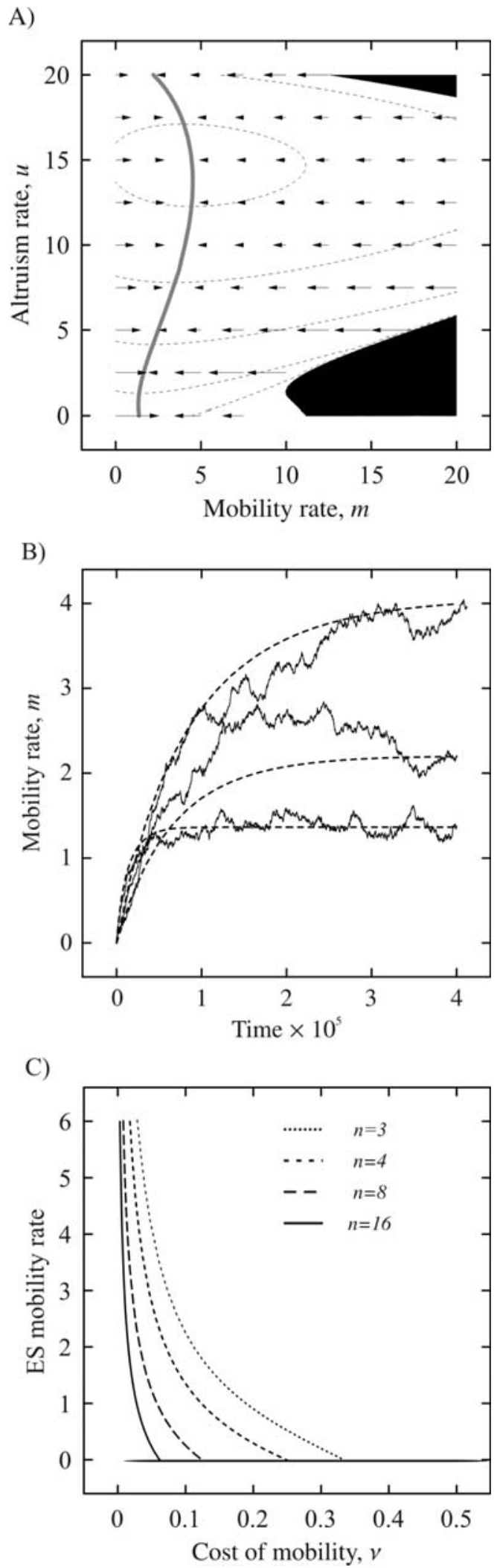
Figure 3
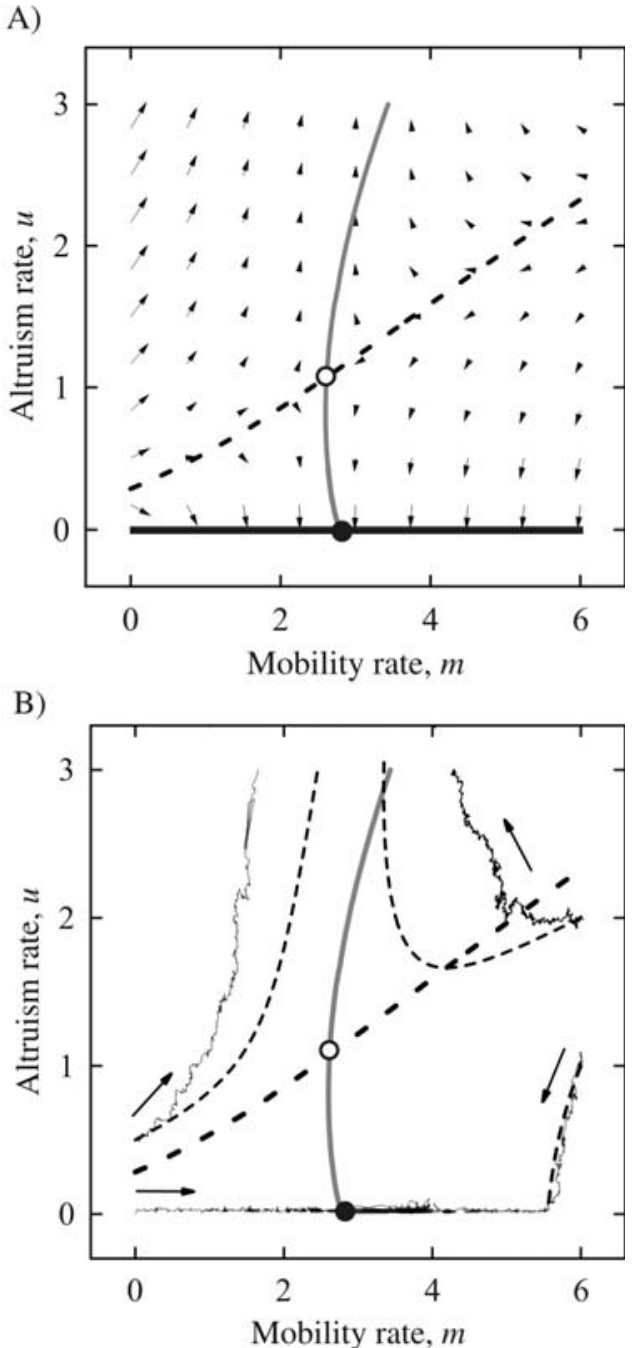

C)

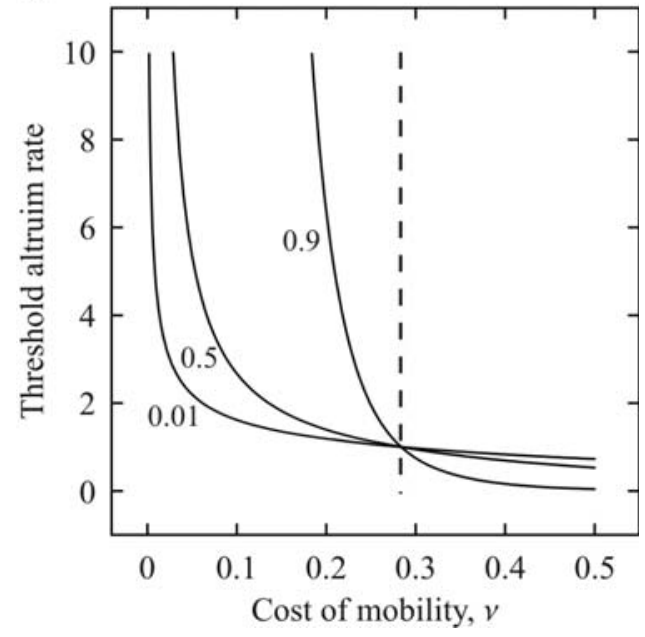


Figure 4

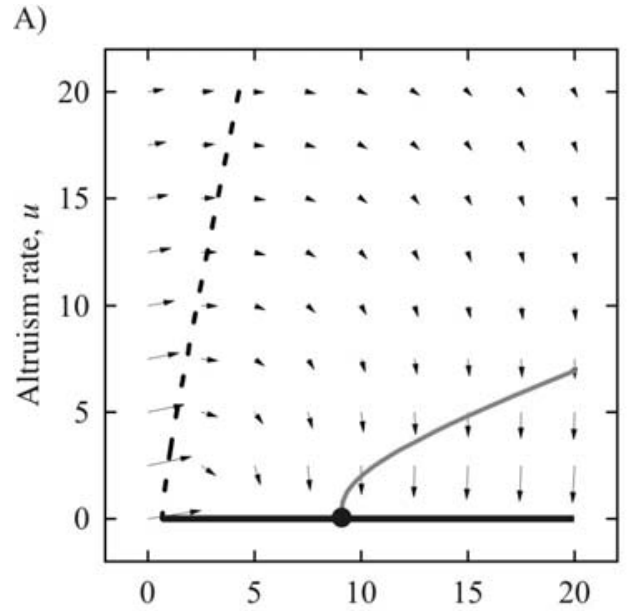

B)

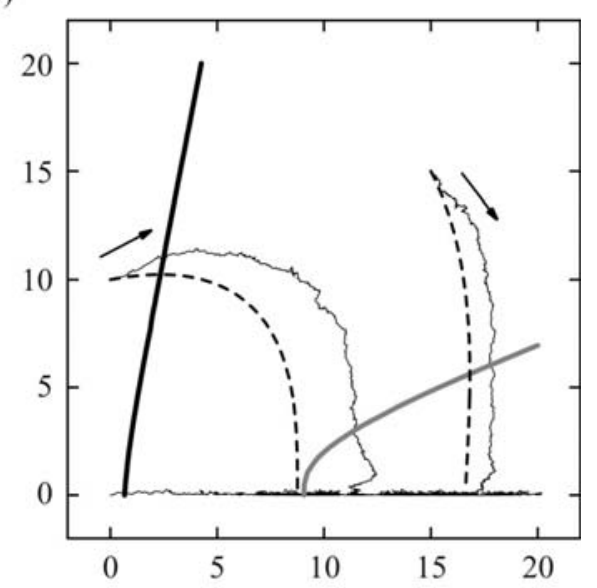

C)

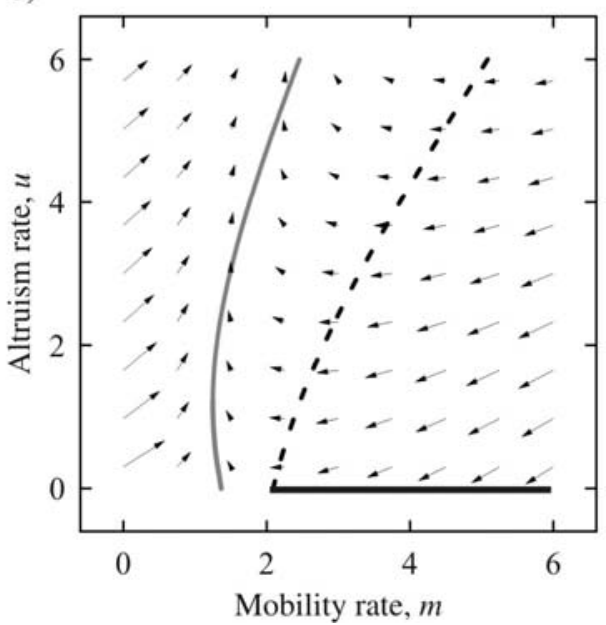

D)

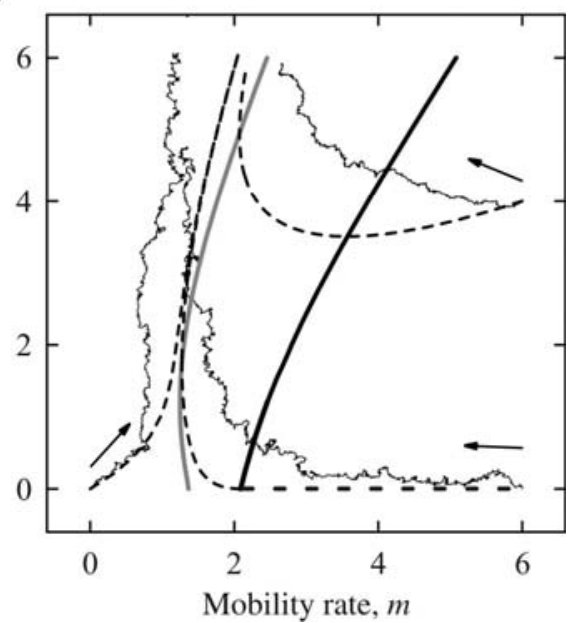


Figure 5
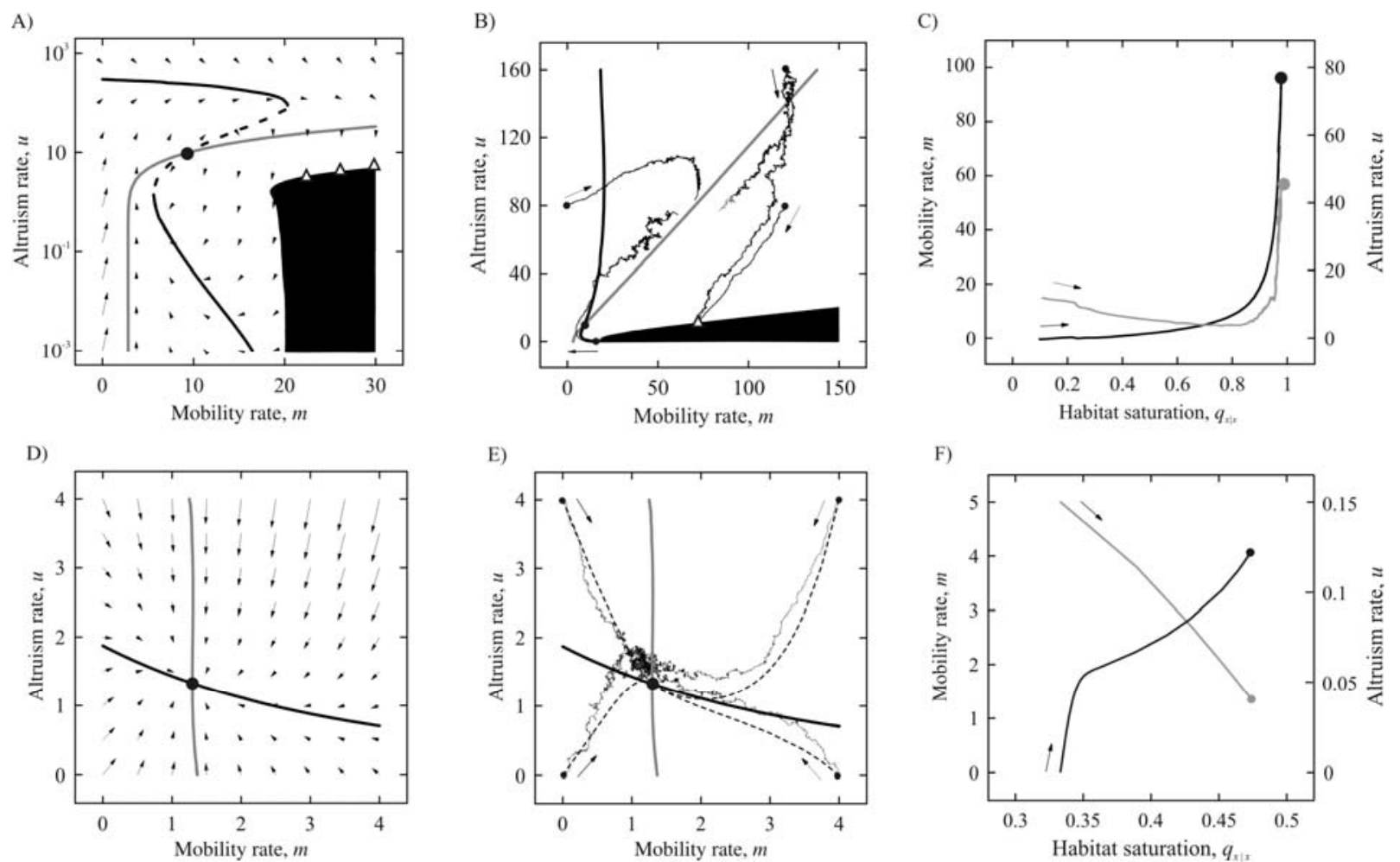
Figure 6

A)

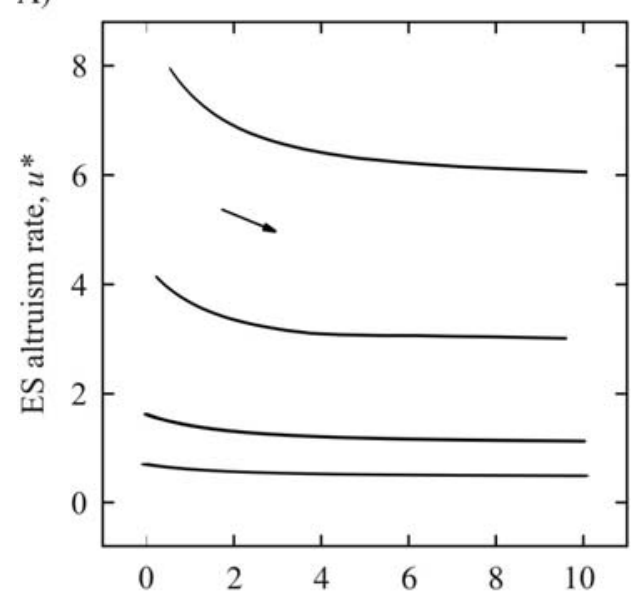

C)

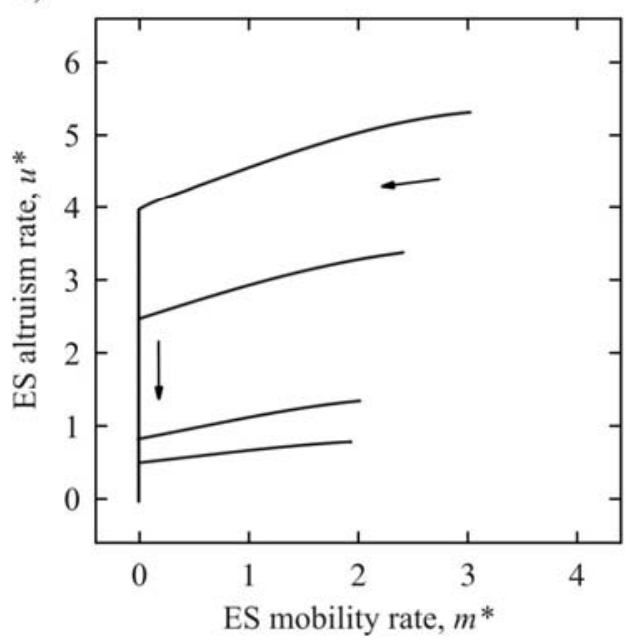

B)

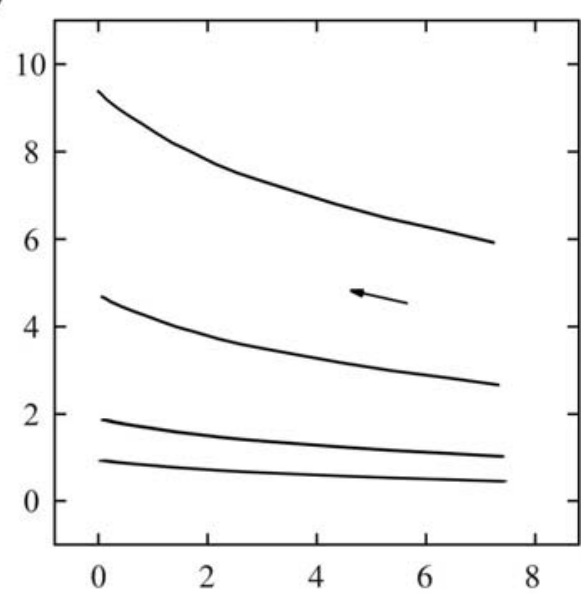

D)

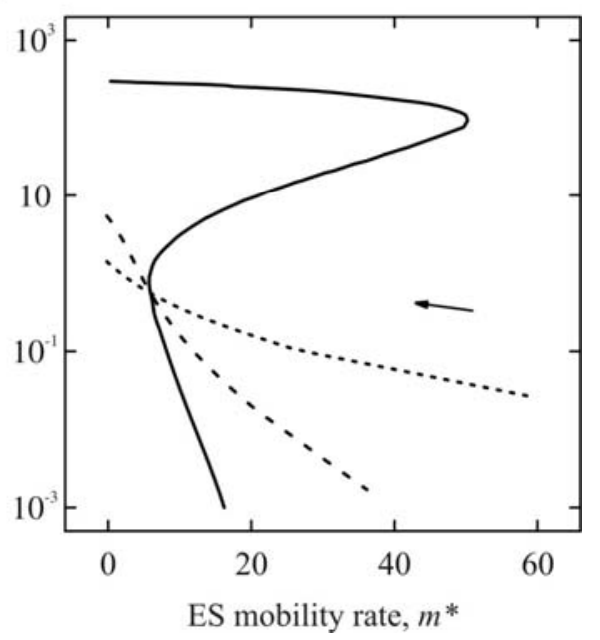

